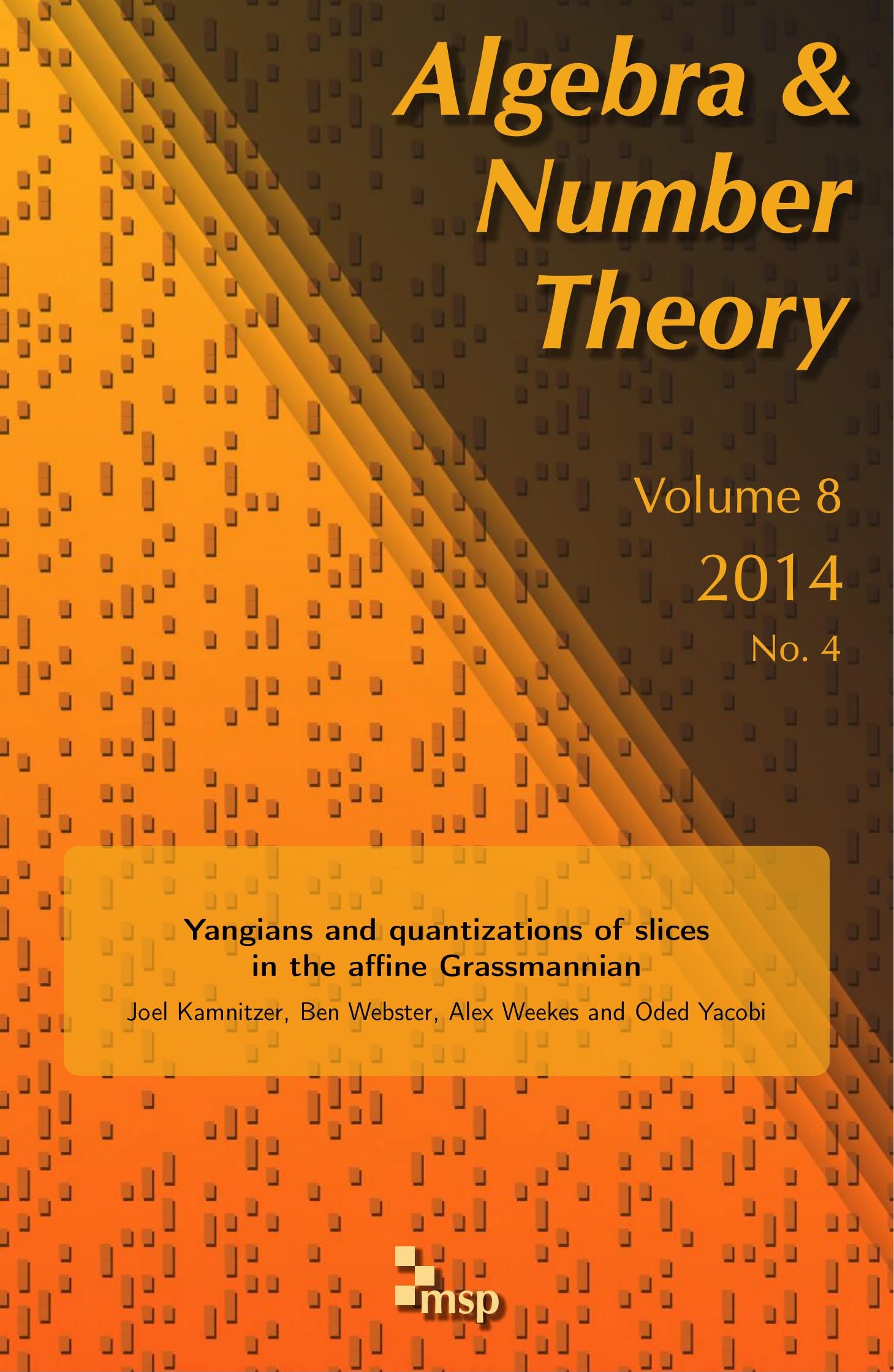




\title{
Yangians and quantizations of slices in the affine Grassmannian
}

\author{
Joel Kamnitzer, Ben Webster, Alex Weekes and Oded Yacobi
}

\begin{abstract}
We study quantizations of transverse slices to Schubert varieties in the affine Grassmannian. The quantization is constructed using quantum groups called shifted Yangians - these are subalgebras of the Yangian we introduce which generalize the Brundan-Kleshchev shifted Yangian to arbitrary type. Building on ideas of Gerasimov, Kharchev, Lebedev and Oblezin, we prove that a quotient of the shifted Yangian quantizes a scheme supported on the transverse slices, and we formulate a conjectural description of the defining ideal of these slices which implies that the scheme is reduced. This conjecture also implies the conjectural quantization of the Zastava spaces for $\mathrm{PGL}_{n}$ of Finkelberg and Rybnikov.
\end{abstract}

\section{Introduction}

We initiate a program which relates the geometry of affine Grassmannians with the representation theory of shifted Yangians. More precisely, we study slices in affine Grassmannians which arise naturally in geometric representation theory; they correspond to weight spaces of irreducible representations under the geometric Satake correspondence. Our main result is that certain subquotients of Yangians quantize these slices.

There is a general program to study symplectic resolutions by means of the representation theory of their quantizations, generalizing the interplay between semisimple Lie algebras and nilpotent cones. We believe that the representation theory of shifted Yangians and its relationship to the geometry of slices in the affine Grassmannian will prove to be a very fruitful area of inquiry.

1A. Slices in the affine Grassmannian. Let $G$ be a complex semisimple group and consider its thick affine Grassmannian $\mathrm{Gr}=G\left(\left(t^{-1}\right)\right) / G[t]$. Attached to each pair of dominant coweights $\lambda \geq \mu$, we have Schubert varieties $\mathrm{Gr}^{\lambda}, \mathrm{Gr}^{\mu} \subset \mathrm{Gr}$, with $\mathrm{Gr}^{\mu} \subset \overline{\mathrm{Gr}^{\lambda}}$. The neighborhood in $\overline{\mathrm{Gr}^{\lambda}}$ of a point in $\mathrm{Gr}^{\mu}$ is encapsulated in a transversal slice to the latter variety in the former, which we denote by $\mathrm{Gr}_{\mu}^{\bar{\lambda}}$. This

Webster was supported by NSF grant DMS-1151473 and by NSA grant H98230-10-1-0199.

MSC2010: primary 20G42; secondary 53D55, 14M15, $14 \mathrm{D} 24$.

Keywords: quantization, affine Grassmannian, quantum groups, Yangian. 
slice is an important object of study in geometric representation theory because under the geometric Satake correspondence it is related to the $\mu$ weight space in the irreducible representation of $G^{\vee}$ of highest weight $\lambda$.

The Manin triple $\left(\mathfrak{g}[t], t^{-1} \mathfrak{g} \llbracket t^{-1} \rrbracket, \mathfrak{g}\left(\left(t^{-1}\right)\right)\right)$ provides Gr with the structure of a Poisson variety. The slice $\mathrm{Gr}_{\mu}^{\bar{\lambda}}$ is an affine Poisson subvariety, and thus its coordinate ring is naturally a Poisson algebra. The purpose of this paper is to explicitly describe quantizations of this Poisson algebra.

1B. Quotients of shifted Yangians. The slice $\mathrm{Gr}_{\mu}^{\bar{\lambda}}$ is defined as the intersection $\overline{\mathrm{Gr}^{\lambda}} \cap \mathrm{Gr}_{\mu}$, where $\mathrm{Gr}_{\mu}$ is an orbit of the group $G_{1} \llbracket t^{-1} \rrbracket$, the first congruence subgroup of $G \llbracket t^{-1} \rrbracket$. Thus on the level of functions $\mathcal{O}\left(\mathrm{Gr}_{\mu}^{\bar{\lambda}}\right)$ is a quotient of $\mathcal{O}\left(\mathrm{Gr}_{\mu}\right)$, and $\mathcal{O}\left(\mathrm{Gr}_{\mu}\right)$ is a subalgebra of $\mathcal{O}\left(G_{1} \llbracket t^{-1} \rrbracket\right)$. In order to quantize $\mathrm{Gr}_{\mu}^{\bar{\lambda}}$ we follow a three-step procedure which mirrors this construction.

We first construct a version $Y$ of the Yangian, which is a subalgebra of the Drinfeld Yangian. Next, we define natural subalgebras $Y_{\mu} \subset Y$, called shifted Yangians, that quantize $\mathrm{Gr}_{\mu}$. This generalizes the shifted Yangian for $\mathfrak{g l}_{n}$ introduced by Brundan and Kleshchev [2006]. Finally, we define a quotient $Y_{\mu}^{\lambda}$ of $Y_{\mu}$ using some remarkable representations of $Y$ as difference operators, constructed by Gerasimov, Kharchev, Lebedev and Oblezin [Gerasimov et al. 2004].

Theorem A. The algebras defined above are all quantizations of the analogous geometric objects. That is:

(1) The Yangian $Y$ quantizes $G_{1} \llbracket t^{-1} \rrbracket$.

(2) The shifted Yangian $Y_{\mu}$ quantizes $\mathrm{Gr}_{\mu}$.

(3) The quotient $Y_{\mu}^{\lambda}$ quantizes a (possibly nonreduced) scheme supported on $\mathrm{Gr}_{\mu}^{\bar{\lambda}}$.

Item (1) is proven using a duality between quantum groups due to Drinfeld and Gavarini, (2) follows simply from (1), and (3) follows using the GerasimovKharchev-Lebedev-Oblezin (GKLO) representation. In fact, we produce a family $Y_{\mu}^{\lambda}(\boldsymbol{c})$ of quantizations which we conjecture to map surjectively to the universal family in the sense of Bezrukavnikov and Kaledin [2004].

Unfortunately, we are not able to prove that the scheme quantized by $Y_{\mu}^{\lambda}$ is reduced. However, we do provide a conjectural description of the generators of the ideal of $\mathrm{Gr}_{\mu}^{\bar{\lambda}}$ inside $\mathrm{Gr}_{\mu}$ and prove that this conjecture implies that $Y_{\mu}^{\lambda}$ quantizes the reduced scheme structure on $\mathrm{Gr}_{\mu}^{\bar{\lambda}}$. Moreover, we prove that this conjecture gives a simple description for the ideal defining $Y_{\mu}^{\lambda}$.

1C. Motivation and relation to other work. Brundan and Kleshchev [2006] construct an isomorphism between quotients of shifted Yangians of $\mathfrak{g l}_{n}$ and $W$-algebras of $\mathfrak{g l}_{m}$. On the one hand, it is known that $W$-algebras are quantizations of Slodowy slices. On the other hand, by the work of Mirković and Vybornov [2007], we 
have an isomorphism between Slodowy slices for $\mathfrak{s l}_{m}$ and slices in the affine Grassmannian for $\mathrm{GL}_{n}$. Thus via these results, we see that quotients of shifted Yangians for $\mathfrak{g l}_{n}$ quantize slices in the affine Grassmannian for $\mathrm{GL}_{n}$. This motivated us to look for a direct construction of quantizations of affine Grassmannian slices (for any semisimple $G$ ) using quotients of shifted Yangians. (The idea that the Brundan-Kleshchev isomorphism should be thought of as a quantization of the Mirković-Vybornov isomorphism was independently observed by Losev [2012, Remark 5.3.4].)

If we take a limit of $\mathrm{Gr}_{\mu}^{\bar{\lambda}}$ as $\lambda \rightarrow \infty$ and $\lambda-\mu$ is fixed, then the slice $\mathrm{Gr}_{\mu}^{\bar{\lambda}}$ becomes the Zastava space $Z_{\lambda-\mu}$. Finkelberg and Rybnikov [2010] have given conjectural quantizations of Zastava spaces (for $\mathrm{PGL}_{n}$ ) using quotients of Borel Yangians, which are a limit of shifted Yangians. We prove that our conjecture about the ideal of $\mathrm{Gr}_{\mu}^{\bar{\lambda}}$ implies the conjecture of Finkelberg and Rybnikov.

Earlier work on shifted Yangians by Brundan and Kleshchev [2008] suggests that one natural direction for future work is the study of a version of category 0 over the algebra $Y_{\mu}^{\lambda}$. Because of the geometric Satake correspondence, we think of category $\mathcal{O}$ for $Y_{\mu}^{\lambda}$ as a categorification of a weight space in a representation of the Langlands dual group $G^{\vee}$. Thus we expect that these categories (with $\lambda$ fixed) carry categorical $\mathfrak{g}^{\vee}$-actions. Moreover, conjectures of Braden, Licata, Proudfoot and Webster [Braden et al. $\geq 2014$ ] suggest that category 0 for $Y_{\mu}^{\lambda}$ should be Koszul dual to similar categories constructed from quiver varieties (in type A, we expect that this reduces to parabolic-singular duality of Beilinson, Ginzburg and Soergel [Beilinson et al. 1996]).

\section{Symplectic structure on slices in the affine Grassmannian}

2A. Notation. For any group $H$, we will write $H\left(\left(t^{-1}\right)\right)=H\left(\mathbb{C}\left(\left(t^{-1}\right)\right)\right)$ for its loop group and write $H[t]=H(\mathbb{C}[t])$ and $H \llbracket t^{-1} \rrbracket=H\left(\mathbb{C} \llbracket t^{-1} \rrbracket\right)$ for its usual subgroups. Let $H_{1} \llbracket t^{-1} \rrbracket$ denote the first congruence subgroup of $H \llbracket t^{-1} \rrbracket$, i.e., the kernel of the evaluation at $t^{-1}=0, H \llbracket t^{-1} \rrbracket \rightarrow H$.

Throughout, $G$ will denote a fixed complex semisimple group with opposite Borel subgroups $B, B_{-}$, unipotent subgroups $N, N_{-}$, maximal torus $T$, Weyl group $W$, set of roots $\Delta$, and simple roots $\left\{\alpha_{i}\right\}_{i \in I}$. We will be concerned with both the coweights and the weights of $G$, which we will be careful to distinguish throughout the paper. Note that the coweights of $G$ are the weights of its Langlands dual group $G^{\vee}$, which we will occasionally consider in this paper.

We write $\left\{\omega_{i}\right\}_{i \in I}$ for the fundamental weights of the simply connected form of $G$.

Following Drinfeld, we use generators $e_{i}, f_{i}, h_{i}$ for $\mathfrak{g}$, where

$$
\left[h_{i}, e_{j}\right]=\left(\alpha_{i}, \alpha_{j}\right) e_{j}, \quad\left[h_{i}, f_{j}\right]=-\left(\alpha_{i}, \alpha_{j}\right) f_{j}, \quad\left[e_{i}, f_{j}\right]=\delta_{i j} h_{i},
$$


along with the usual Serre relations. Let $\left(a_{i j}\right)_{1 \leq i, j \leq n}$ be the Cartan matrix of $\mathfrak{g}$, and let $d_{i}$ be the unique coprime positive integers such that $b_{i j}=d_{i} a_{i j}$ is a symmetric matrix. Then the associated invariant form on $\mathfrak{g}$ is defined by $\left(e_{i}, f_{j}\right)=\delta_{i j}$ and $\left(\alpha_{i}, \alpha_{j}\right)=\left(h_{i}, h_{j}\right)=d_{i} a_{i j}$, and in particular, $h_{i}$ is the image of $\alpha_{i}$ under the identification of $\mathfrak{h}$ and $\mathfrak{h}^{*}$.

This is as opposed to the standard Chevalley generators $e_{i}^{\prime}, f_{i}^{\prime}, h_{i}^{\prime}$, which we will identify as

$$
e_{i}=-d_{i}^{1 / 2} e_{i}^{\prime}, \quad f_{i}=-d_{i}^{1 / 2} f_{i}^{\prime}, \quad h_{i}=d_{i} h_{i}^{\prime} .
$$

In this way we have fundamental weights $\omega_{i}\left(h_{j}^{\prime}\right)=\delta_{i j}$ and a lift of the Weyl group defined via $\overline{s_{i}}=\exp \left(f_{i}^{\prime}\right) \exp \left(-e_{i}^{\prime}\right) \exp \left(f_{i}^{\prime}\right)$.

If $\mu$ is a weight or coweight, we write $\mu^{*}=-w_{0} \mu$. Likewise, we write $i^{*}$ if $\alpha_{i^{*}}=-w_{0} \alpha_{i}$.

Let $V$ be a representation of $G$, and let $v \in V, \beta \in V^{*}$. The matrix entry $\Delta_{\beta, v}$ is a function on $G$ given by $\Delta_{\beta, v}(g)=\langle\beta, g v\rangle$. If $w_{1}, w_{2} \in W$ and $\tau$ is a dominant weight, we define

$$
\Delta_{w_{1} \tau, w_{2} \tau}(g)=\left\langle\overline{w_{1}} v_{-\tau}, \overline{w_{2}} v_{\tau}\right\rangle
$$

using the lift described above, where $v_{\tau}$ is the highest-weight vector for the irreducible representation $V(\tau)$ and $v_{-\tau}$ is the dual lowest-weight vector in $V\left(\tau^{*}\right)$.

Using this matrix entry (also known as a generalized minor), we define the function $\Delta_{\beta, v}^{(s)}$ on $G\left(\left(t^{-1}\right)\right)$, for $s \in \mathbb{Z}$, whose value at $g$ is the coefficient of $t^{-s}$ in the polynomial $\Delta_{\beta, v}(g)$. So we have the formula

$$
\Delta_{\beta, v}(g)=\sum_{s=-\infty}^{\infty} \Delta_{\beta, v}^{(s)}(g) t^{-s} .
$$

2B. Slices in the affine Grassmannian. Let $G$ be a semisimple complex group. In this paper, we will work with the thick affine Grassmannian $\mathrm{Gr}=G\left(\left(t^{-1}\right)\right) / G[t]$. We have an embedding of the usual thin affine Grassmannian into the thick affine Grassmannian

$$
G((t)) / G \llbracket t \rrbracket \cong G\left[t, t^{-1}\right] / G[t] \hookrightarrow G\left(\left(t^{-1}\right)\right) / G[t] .
$$

We work with the thick affine Grassmannian since it is forced upon us by the noncommutative algebras we consider. One manifestation of this is that the thick Grassmannian is an honest scheme, while the thin Grassmannian is only an indscheme. However, at a first reading, this difference will be of little importance, and the reader can pretend that we are working with the usual thin affine Grassmannian.

Any coweight $\lambda$ can be thought of as a $\mathbb{C}\left[t, t^{-1}\right]$-point of $G$, which we can think of as a $\mathbb{C}\left(\left(t^{-1}\right)\right)$-point as well. To avoid confusion, we use $t^{\lambda}$ to denote this point in $G\left(\left(t^{-1}\right)\right)$. We also use $t^{\lambda}$ for the image of $t^{\lambda}$ in Gr. 
Let $\lambda$ and $\mu$ denote dominant coweights. Define

$$
\mathrm{Gr}^{\lambda}=G[t] t^{\lambda}, \quad \mathrm{Gr}_{\mu}=G_{1} \llbracket t^{-1} \rrbracket t^{w_{0} \mu} .
$$

Recall that the thin affine Grassmannian is precisely $\bigcup_{\lambda} G r^{\lambda}$.

Our main object of interest will be

$$
\mathrm{Gr}_{\mu}^{\bar{\lambda}}:=\overline{\mathrm{Gr}^{\lambda}} \cap \mathrm{Gr}_{\mu}
$$

This variety is a transverse slice to $\mathrm{Gr}^{\mu}$ inside of $\overline{\mathrm{Gr}^{\lambda}}$ since $\mathrm{Gr}_{\mu}$ intersects every $\mathrm{Gr}^{\nu}$ transversely and the intersection $\mathrm{Gr}_{\mu}^{\bar{\mu}}$ is just the point $t^{w_{0} \mu}$. In particular, this variety is nonempty if and only if $\mu \leq \lambda$, that is, if $\mathrm{Gr}^{\mu} \subset \overline{\mathrm{Gr}^{\lambda}}$. These varieties arise naturally under the geometric Satake correspondence of Lusztig [1983], Ginzburg [1995], and Mirković and Vilonen [2007]: the intersection homology of $\mathrm{Gr}_{\mu}^{\bar{\lambda}}$ is identified with the $\mu$-weight space of the irreducible $G^{\vee}$-representation of highest weight $\lambda$.

Note that $\mathbb{C}^{\times}$acts on $G r$ by loop rotation. This action preserves the $G[t]$ and $G_{1} \llbracket t^{-1} \rrbracket$ orbits, and so $\mathbb{C}^{\times}$acts on $\mathrm{Gr}_{\mu}^{\bar{\lambda}}$. The following result is standard (it is a special case of general results about flag varieties and their big cells).

Proposition 2.1. (1) $\mathrm{Gr}_{\mu}^{\bar{\lambda}}$ is an affine variety of dimension $2\langle\rho, \lambda-\mu\rangle$.

(2) The action of $\mathbb{C}^{\times}$on $\mathrm{Gr}_{\mu}^{\bar{\lambda}}$ contracts $\mathrm{Gr}_{\mu}^{\bar{\lambda}}$ to the unique fixed point $t^{w_{0} \mu}$.

Example 2.2. If $\lambda=\mu+\alpha_{i}^{\vee}$, then $\operatorname{Gr}_{\mu}^{\bar{\lambda}}$ is isomorphic to the Kleinian singularity $\mathbb{C}^{2} /(\mathbb{Z} / n+2)$, where $n=\left\langle\mu, \alpha_{i}\right\rangle$. To see this, first we identify

$$
\mathbb{C}^{2} /(\mathbb{Z} / n+2)=\left\{(u, v, w) \mid u v+w^{n+2}=0\right\},
$$

and then we define the isomorphism

$$
\begin{aligned}
\mathbb{C}^{2} /(\mathbb{Z} / n+2) & \rightarrow \operatorname{Gr}_{\mu}^{\bar{\lambda}}, \\
(u, v, w) & \mapsto \phi_{i}\left(\left[\begin{array}{cc}
1-w t^{-1} & v t^{-(n+1)} \\
u t^{-1} & 1+w t^{-1}+\cdots+w^{n+1} t^{-(n+1)}
\end{array}\right]\right) t^{w_{0} \mu},
\end{aligned}
$$

where $\phi_{i}: \mathrm{SL}_{2} \rightarrow G$ denotes the $\mathrm{SL}_{2}$ corresponding to $\alpha_{i}$.

Let $G\left(\left(t^{-1}\right)\right)_{\mu}$ denote the stabilizer of $t^{w_{0} \mu}$ inside of $G\left(\left(t^{-1}\right)\right)$. The following easy result describes the stabilizer on the Lie algebra level.

Lemma 2.3. $\operatorname{Lie}\left(G\left(\left(t^{-1}\right)\right)_{\mu}\right)=\mathfrak{t}[t] \oplus \bigoplus_{\alpha \in \Delta} t^{\left\langle\alpha, w_{0} \mu\right\rangle} \mathfrak{g}_{\alpha}[t]$.

Proof. The result follows immediately after observing that for $g \in G\left(\left(t^{-1}\right)\right)$, we have $g \in G\left(\left(t^{-1}\right)\right)_{\mu}$ if and only if $t^{-w_{0} \mu} g t^{w_{0} \mu} \in G[t]$.

In what follows, we will need the following set-theoretic description of $\overline{\mathrm{Gr}^{\lambda}}$ due to Finkelberg and Mirković [1999, (10.2)]. As we shall see, it is much trickier to find a description of this variety with its natural reduced scheme structure. 
Proposition 2.4. Let $g \in G\left(\left(t^{-1}\right)\right)$. We have $[g] \in \overline{\mathrm{Gr}^{\lambda}}$ if and only if $\Delta_{\beta, v}^{(s)}(g)=0$ for all dominant weights $\tau$, for all $v \in V(\tau), \beta \in V(\tau)^{*}$, and for all $s<\left\langle\lambda, w_{0} \tau\right\rangle$.

Proof. Fix $\tau$ and let $k$ be the minimal $s$ such that there exist $\beta \in V(\tau)^{*}, v \in V(\tau)$ with $\Delta_{\beta, v}^{(s)}(g) \neq 0$ (if such a minimum exists). It is easy to see that $k$ only depends on the $G[t]$ double coset containing $g$. Thus if $[g] \in \mathrm{Gr}^{\lambda}$, we have that $k=\left\langle\lambda, w_{0} \tau\right\rangle$. The result follows.

The proof makes it clear that the Proposition holds even if $\tau$ only ranges over a set of dominant weights which spans (over $\mathbb{Q}$ ) the weight lattice.

2C. Symplectic structure on the affine Grassmannian. There is a nondegenerate pairing on $\mathfrak{g}\left(\left(t^{-1}\right)\right)$ coming from residue and the invariant form on $\mathfrak{g}$. Hence the Lie algebras $\mathfrak{g}[t], t^{-1} \mathfrak{g} \llbracket t^{-1} \rrbracket$, and $\mathfrak{g}\left(\left(t^{-1}\right)\right)$ form a Manin triple (see [Drinfeld 1987, Example 3.3]). This induces a Poisson-Lie structure on $G\left(\left(t^{-1}\right)\right)$ with $G[t]$ and $G_{1} \llbracket t^{-1} \rrbracket$ as Poisson subgroups. In particular, it coinduces a Poisson structure on $\mathrm{Gr}$, by standard calculations which date back to work of Drinfeld [1993].

Let us state a couple of results concerning the interaction between this symplectic structure and the geometry considered in the previous section. These results were originally obtained by Mirković (personal communication).

Theorem 2.5. Subvarieties $\mathrm{Gr}_{\mu}^{\lambda}=\mathrm{Gr}^{\lambda} \cap \mathrm{Gr}_{\mu}$ are symplectic leaves of $\mathrm{Gr}$.

Proof. First we note that $\mathrm{Gr}_{\mu}^{\lambda}$ are connected by [Richardson 1992, 1.4], since $\mathfrak{g}\left(\left(t^{-1}\right)\right)=\mathfrak{g}[t] \oplus t^{-1} \mathfrak{g} \llbracket t^{-1} \rrbracket$. The argument is stated there for finite-dimensional groups, but carries through to the loop situation without issues. Then the result follows from [Lu and Yakimov 2008, Corollary 2.9].

These are not all symplectic leaves of $\mathrm{Gr}$, since not every $G_{1} \llbracket t^{-1} \rrbracket$-orbit contains a point $t^{w_{0} \mu}$ and not every $G[t]$-orbit contains a point $t^{\lambda}$. A general symplectic leaf which lies in the thin affine Grassmannian is of the form $\mathrm{Gr}^{\lambda} \cap G_{1} \llbracket t^{-1} \rrbracket g t^{w_{0} \mu}$, where $g \in G$.

Let $S^{\mu}=N\left(\left(t^{-1}\right)\right) t^{w_{0} \mu}$. An $M V$ cycle is a component of $\overline{\mathrm{Gr}^{\lambda}} \cap S^{\mu}$. By MirkovićVilonen, these MV cycles give a basis for weight spaces of irreducible representations of the Langlands dual group. As we now see, the MV cycles are Lagrangians in $\mathrm{Gr}_{\mu}^{\bar{\lambda}}$.

\section{Proposition 2.6. $\overline{\mathrm{Gr}^{\lambda}} \cap S^{\mu}$ is a Lagrangian subvariety of $\mathrm{Gr}_{\mu}^{\bar{\lambda}}$.}

Proof. First we prove that $\overline{\mathrm{Gr}^{\lambda}} \cap S^{\mu} \subset \mathrm{Gr}_{\mu}^{\bar{\lambda}}$. Since $N$ is unipotent, we have that $N\left(\left(t^{-1}\right)\right)=N_{1} \llbracket t^{-1} \rrbracket N[t]$. Now by Lemma 2.3, we have that $N[t] t^{w_{0} \mu}=t^{w_{0} \mu}$. Hence $N\left(\left(t^{-1}\right)\right) t^{w_{0} \mu}=N_{1} \llbracket t^{-1} \rrbracket t^{w_{0} \mu}$ and thus $S^{\mu} \subset \mathrm{Gr}_{\mu}$.

From [Mirković and Vilonen 2007, Theorem 3.2], $\operatorname{dim} \overline{\mathrm{Gr}^{\lambda}} \cap S^{\mu}=\langle\rho, \lambda-\mu\rangle$, and thus the intersection $\overline{\mathrm{Gr}^{\lambda}} \cap S^{\mu}$ is half-dimensional in $\operatorname{Gr}_{\mu}^{\bar{\lambda}}$. Hence it is Lagrangian if and only if it is coisotropic. The variety $\operatorname{Gr}_{\mu}^{\bar{\lambda}}$ is affine, and so it suffices to check that 
the Poisson bracket of any two functions that vanish on $\overline{\mathrm{Gr}^{\lambda}} \cap S^{\mu}$ vanishes there as well. The functions vanishing on $S^{\mu} \cap \overline{\mathrm{Gr}^{\lambda}}$ are generated by all functions of negative weight under the action of the coweight $\rho^{\vee}: \mathbb{C}^{\times} \rightarrow G$. Since that action preserves the Poisson structure, the Poisson bracket of two negative weight functions is again of negative weight; this completes the proof.

It is natural to ask whether $\operatorname{Gr}_{\mu}^{\bar{\lambda}}$ has a symplectic resolution. Let us temporarily assume that $G$ is of adjoint type and let us fix a sequence $\vec{\lambda}=\left(\lambda_{1}, \ldots, \lambda_{n}\right)$ of fundamental coweights such that $\lambda=\lambda_{1}+\cdots+\lambda_{n}$. (If we do not assume that $G$ is of adjoint type, then we may not be able to write $\lambda$ as a sum of fundamental coweights of $G$.) Then we have the open and closed convolutions

$$
G r^{\vec{\lambda}}:=G r^{\lambda_{1}} \tilde{\times} \cdots \tilde{\times} G r^{\lambda_{n}}, \quad \overline{G r^{\vec{\lambda}}}:=\overline{G r^{\lambda_{1}}} \tilde{\times} \cdots \tilde{\times} \overline{G^{\lambda_{n}}}
$$

along with the convolution morphisms $m: \mathrm{Gr}^{\vec{\lambda}} \rightarrow \overline{\mathrm{Gr}^{\lambda}}$ and $\bar{m}: \overline{\mathrm{Gr}^{\vec{\lambda}}} \rightarrow \overline{\mathrm{Gr}^{\lambda}}$. (Here the convolution $A \tilde{\times} B$ of two $G[t]$-invariant subsets $A, B$ in $\mathrm{Gr}$ is defined by $p^{-1}(A) \times_{G[t]} B$, where $p: G\left(\left(t^{-1}\right)\right) \rightarrow$ Gr. $)$

Let

$$
\mathrm{Gr}_{\mu}^{\vec{\lambda}}:=m^{-1}\left(\mathrm{Gr}_{\mu}\right), \quad \mathrm{Gr}_{\mu}^{\overrightarrow{\vec{\lambda}}}:=\bar{m}^{-1}\left(\mathrm{Gr}_{\mu}\right) .
$$

Recall that a normal variety $X$ with a fixed symplectic structure $\Omega$ on its smooth locus is said to have symplectic singularities if, locally on $X$, there are resolutions of singularities $p: U \rightarrow X$, where $p^{*} \Omega$ is the restriction of a closed 2-form on $U$ (which is not assumed to be nondegenerate on the exceptional locus).

A variety $X$ is said to have terminal singularities if there is a resolution of singularities of $X$ such that each irreducible exceptional fiber has positive discrepancy, that is, $X$ is as close to being smoothly resolved as is crepantly possible. A terminalization $X \rightarrow Y$ is a map which is birational, proper, and crepant with $X$ having terminal singularities. We say a variety $X$ is $\mathbb{Q}$-factorial if every Weil divisor on $X$ has an integer multiple which is Cartier.

Theorem 2.7. The variety $\mathrm{Gr}_{\mu}^{\bar{\lambda}}$ has symplectic singularities, and $\mathrm{Gr} \mathrm{r}_{\mu}^{\bar{\lambda}}$ is a $\mathbb{Q}$-factorial terminalization of $\mathrm{Gr}_{\mu}^{\bar{\lambda}}$.

Proof. First, we claim that $\operatorname{Gr} \underset{\mu}{\vec{\lambda}}$ has singular locus in codimension at least 4. Since $\mathrm{Gr}_{\mu}$ is transverse to every $G[t]$-orbit, the codimension of the singular locus cannot jump when we pass to $\mathrm{Gr} \underset{\mu}{\vec{\lambda}}$, so we need only establish the same result for $\mathrm{Gr}_{\mathrm{r}}^{\overrightarrow{\bar{\lambda}}}$, for which it suffices to consider the case of a fundamental coweight. If $\omega_{i}$ is a fundamental coweight and $v$ is a dominant coweight such that $\mathrm{Gr}^{v} \subset \mathrm{Gr}^{\overline{\omega_{i}}}$, then we have that $\rho^{\vee}\left(\omega_{i}-v\right) \geq 2$, since $\omega_{i}-\alpha_{j}$ is never dominant. Thus, the singular locus $\mathrm{Gr}^{\nu}$ has codimension at least 4 .

As Beauville [2000, (1.2)] notes, since $\mathrm{Gr} \mathrm{r}_{\mu}^{\overrightarrow{\hat{\lambda}}}$ is regular in codimension 3 and normal, the existence of a symplectic form on its smooth locus implies that it has 
symplectic singularities. Since we have a Poisson map $\mathrm{Gr}_{\mu}^{\bar{\lambda}} \rightarrow \mathrm{Gr}_{\mu}^{\bar{\lambda}}$, this variety also has symplectic singularities. By a result of Namikawa [2001, Corollary 1], this regularity in codimension 3 also implies that $\mathrm{Gr}_{\mu}^{\overrightarrow{\vec{\lambda}}}$ is terminal.

Because each local singularity in $\mathrm{Gr}_{\mu}^{\bar{\lambda}}$ is a local singularity in $\mathrm{Gr}$, $\overline{\bar{\lambda}}$, and these are the product of local singularities in $\mathrm{Gr}^{\frac{\mu}{\omega_{i}}}$, we need only prove $\mathbb{Q}$-factoriality in this case. The group of Weil divisors of $\mathrm{Gr}^{\overline{\omega_{i}}}$ is the same as that of $\mathrm{Gr}^{\omega_{i}}$, which is an affine bundle over $G / P_{i}$, where $P_{i}$ is the maximal parabolic containing all negative simple root spaces but $\mathfrak{g}_{-\alpha_{i}}$. Thus, the Weil divisor group of $G / P_{i}$ is isomorphic to $\mathbb{Z}$.

Since $\mathrm{Gr}^{\overline{\omega_{i}}}$ is projective, some Weil divisor on $\mathrm{Gr}^{\overline{\omega_{i}}}$ is Cartier. Thus, the group generated by any nontrivial Weil divisor must intersect the image of the Cartier divisors, and so $\mathrm{Gr}^{\overline{\omega_{i}}}$ is $\mathbb{Q}$-factorial. ${ }^{1}$

By general properties of Schubert varieties and Bott-Samuelson resolutions, the map $\mathrm{Gr}_{\mu}^{\bar{\lambda}} \rightarrow \mathrm{Gr}_{\mu}^{\bar{\lambda}}$ is proper and birational. The preimage of $\mathrm{Gr}^{\mu}$ for $\mu \neq \lambda, \lambda-\alpha_{i}$ has codimension at least 4 , so any exceptional divisor must be the closure of a component of the preimage of $\mathrm{Gr}^{\lambda-\alpha_{i}}$. The coefficients of these divisors in the discrepancy can thus be computed locally in a neighborhood of $x \in \mathrm{Gr}^{\lambda-\alpha_{i}}$, but the germ of the map is equivalent to the minimal resolution of a Kleinian singularity by Example 2.2. The Kleinian singularities are known to be crepant.

An obvious question is when $\mathrm{Gr}_{\mu}^{\bar{\lambda}}$ has a symplectic resolution. First, we make the following conjecture.

Conjecture 2.8. Any symplectic resolution of $\mathrm{Gr}_{\mu}^{\bar{\lambda}}$ is of the form $\mathrm{Gr}_{\mu}^{\overline{\vec{\lambda}}}$.

We can easily see when $\mathrm{Gr}_{\mu}^{\overline{\vec{\lambda}}}$ is actually a resolution.

Theorem 2.9. The following are equivalent.

(1) $\mathrm{Gr}_{\mu}^{\bar{\lambda}}$ possesses a symplectic resolution of singularities.

(2) $\mathrm{Gr}_{\mu}^{\bar{\lambda}}$ is smooth and thus is a symplectic resolution of singularities of $\mathrm{Gr}_{\mu}^{\bar{\lambda}}$.

(3) $\mathrm{Gr}_{\mu}^{\vec{\lambda}}=\mathrm{Gr}_{\mu} \overrightarrow{\bar{\lambda}}$.

(4) There do not exist coweights $v_{1}, \ldots, v_{n}$ such that $v_{1}+\cdots+v_{n}=\mu$; for all $k$, $v_{k}$ is a weight of $V\left(\lambda_{k}\right)$; and for some $k, v_{k}$ is a not an extremal weight of $V\left(\lambda_{k}\right)$ (here we regard the $v_{k}$ as weights of $G^{\vee}$ ).

Proof. (1) $\Longrightarrow$ (2): If $\mathrm{Gr}_{\mu}^{\bar{\lambda}}$ has a symplectic resolution, then by [Namikawa 2011, 5.6], any $\mathbb{Q}$-factorial terminalization of $\mathrm{Gr}_{\mu}^{\bar{\lambda}}$, in particular $\mathrm{Gr}_{\mu}^{\overrightarrow{\vec{\lambda}}}$, is smooth.

${ }^{1}$ We thank Alexander Braverman for suggesting this portion of the argument to us. 
$(2) \Longrightarrow(1)$ : In this case, $\operatorname{Gr} \underset{\mu}{\overline{\vec{\lambda}}}$ is an example of a symplectic resolution of singularities. $(2) \Rightarrow(3)$ : By [Evens and Mirković 1999, Theorem 0.1(b)], the smooth locus of $\overline{\mathrm{Gr}^{\lambda}}$ is precisely $\mathrm{Gr}^{\lambda}$. Thus the smooth locus of $\mathrm{Gr}^{\bar{\lambda}}$ is precisely $\mathrm{Gr}^{\vec{\lambda}}$.

Next we assume that there is a point $x$ in $\mathrm{Gr}_{\mu}^{\overrightarrow{\bar{\lambda}}}$ not in $\mathrm{Gr}_{\mu}^{\vec{\lambda}}$; we know that $\mathrm{Gr}$ is not smooth at $x$. By the transversality of the $G_{1} \llbracket t^{-1} \rrbracket$ and $G[t]$ orbits, the completion of $\mathrm{Gr} \overline{\bar{\lambda}}$ at $x$ coincides with the completion of $\mathrm{Gr}_{\mu}^{\overline{\vec{\lambda}}}$ at $x$ times something smooth. Therefore $\mathrm{Gr} \underset{\mu}{\vec{\lambda}}$ cannot be smooth at $x$ either.

(3) $\Longrightarrow(2)$ : Clear.

(3) $\Longrightarrow$ (4): If there exist $v_{1}, \ldots, v_{n}$ as in (3), then $\left(t^{\nu_{1}}, t^{\nu_{1}+\nu_{2}}, \ldots, t^{\mu}\right) \in \mathrm{Gr}_{\mu}^{\vec{\lambda}} \backslash \mathrm{Gr}_{\mu}^{\vec{\lambda}}$.

(4) $\Longrightarrow(3)$ : Suppose that there exists

$$
\left(L_{1}, \ldots, L_{n}\right) \in \mathrm{Gr}_{\mu}^{\overrightarrow{\bar{\lambda}}} \backslash \mathrm{Gr}_{\mu}^{\vec{\lambda}} .
$$

Recall that we have a $\mathbb{C}^{\times} \times T$ action on $\mathrm{Gr}$ where the first factor acts by loop rotation. Consider a map $\mathbb{C}^{\times} \rightarrow \mathbb{C}^{\times} \times T$ which is the identity into the first factor and a generic dominant coweight into the second factor. We get a resulting $\mathbb{C}^{\times}$ action on $\mathrm{Gr}$ whose attracting sets are the $I_{-}$orbits, where $I_{-}$is the preimage of $B$ under $G \llbracket t^{-1} \rrbracket \rightarrow G$.

Let

$$
\left(t^{\mu_{1}}, \ldots, t^{\mu_{n}}\right)=\lim _{s \rightarrow 0} s \cdot\left(L_{1}, \ldots, L_{n}\right) .
$$

From the definition of $\mathrm{Gr}_{\mu}^{\vec{\lambda}}$, we see that $\mu_{n}=\mu$. Also, for each $k$, we see that $d\left(t^{\mu_{k-1}}, t^{\mu_{k}}\right) \leq \lambda_{k}$ (where $d$ denotes the dominant coweight valued distance function on Gr), and so $v_{k}:=\mu_{k}-\mu_{k-1}$ is a weight of $V\left(\lambda_{k}\right)$. Thus we obtain $v_{1}, \ldots, v_{n}$ with $v_{1}+\cdots+v_{n}=\mu$. Moreover, since $\left(L_{1}, \ldots, L_{n}\right) \notin \mathrm{Gr}_{\mu}^{\vec{\lambda}}$, we have $d\left(L_{k-1}, L_{k}\right)<\lambda_{k}$ for some $k$, and so $v_{k}$ is a nonextremal weight of $V\left(\lambda_{k}\right)$.

If $\lambda$ is a sum of minuscule coweights, then the above conditions hold. For any simple $G$ not of type A, there are nonminiscule fundamental coweights $\lambda$; for such $\lambda$, we can choose $\mu$ such that the above conditions do not hold. So there exist $\operatorname{Gr}_{\mu}^{\bar{\lambda}}$ which do not admit symplectic resolutions.

2D. Beilinson-Drinfeld Grassmannian. Using the Beilinson-Drinfeld Grassmannian, we can define a family of Poisson varieties over $\mathbb{A}^{n}$ whose special fiber is $\mathrm{Gr}_{\mu}^{\bar{\lambda}}$. In this work, this family will only be used as motivation for a similar family of quantizations of $\mathrm{Gr}_{\mu}^{\bar{\lambda}}$; as illustrated in works such as [Bezrukavnikov and Kaledin 2004; Braden et al. 2012; Losev 2012], the universal symplectic deformation of a symplectic singularity as a symplectic variety is intimately tied to understanding its quantizations (see Section 4D). From this perspective, a natural next step (beyond the scope of this paper) would be to study quantizations of the total spaces of these deformations, not just of a single fiber. 
Recall that we have the moduli interpretation of the affine Grassmannian (see [Mirković and Vilonen 2007, Section 5])

$\mathrm{Gr}=\left\{(E, \phi) \mid E\right.$ is a principal $G$-bundle on $\mathbb{P}^{1}$

and $\phi:\left.\left.E\right|_{\mathbb{P}^{1} \backslash\{0\}} \rightarrow E^{0}\right|_{\mathbb{P}^{1} \backslash\{0\}}$ is an isomorphism $\}$,

where $E^{0}$ denotes the trivial $G$-bundle. We say that $(E, \phi)$ has Hecke type $\lambda$ at 0 if $(E, \phi)$ gives a point in $\mathrm{Gr}^{\lambda}$ under the above identification.

Note that the action of $G \llbracket t^{-1} \rrbracket$ by left multiplication in the homogeneous space definition becomes change of trivialization in the new definition. Thus the $G \llbracket t^{-1} \rrbracket$ orbit of $(E, \phi)$ is determined by isomorphism class of the $G$-bundle $E$, which is given by a dominant coweight. Note also that the action of $G_{1} \llbracket t^{-1} \rrbracket$ corresponds to changes of trivialization which do not change anything at $\infty$.

Let $\mu$ be a dominant coweight and let $P$ be the corresponding standard parabolic subgroup (so that $W_{P}$ is the stabilizer of $\mu$ in the Weyl group). Let $E$ be a principal $G$-bundle of type $\mu$. Then $E$ has a canonical $P$-structure.

Now let $(E, \phi) \in \mathrm{Gr}$. Let $\mu$ be the isomorphism type of $E$. Then $\phi_{\infty}$ carries the parabolic structure at $\infty$ to a parabolic subgroup of $G$ of type $\mu$. Hence we see that the $G_{1} \llbracket t^{-1} \rrbracket$ orbits on $\mathrm{Gr}$ are labeled by a pair consisting of a dominant coweight $\mu$ and a parabolic subgroup of $G$ of type $\mu$. In particular, $\mathrm{Gr}_{\mu}$ is the locus of those ( $E, \phi)$ where $E$ has isomorphism type $\mu$ and the parabolic subgroup produced is the standard one.

We now consider the Beilinson-Drinfeld deformation of the affine Grassmannian. This is a family $\mathrm{Gr}_{\mathbb{A}^{n}}$ over $\mathbb{A}^{n}$ whose fiber at $a_{1}, \ldots, a_{n} \in \mathbb{A}^{n}$ is given as

$\mathrm{Gr}_{a_{1}, \ldots, a_{n}}=\left\{(E, \phi) \mid E\right.$ is a principal $G$-bundle on $\mathbb{P}^{1}$ and $\phi:\left.\left.E\right|_{\mathbb{P}^{1} \backslash\left\{a_{1}, \ldots, a_{n}\right\}} \rightarrow E^{0}\right|_{\mathbb{P}^{1} \backslash\left\{a_{1}, \ldots, a_{n}\right\}}$ is an isomorphism $\}$.

Let $\mathrm{Gr}_{\mu, \mathrm{A}^{n}}$ be the locus of $(E, \phi)$, where $E$ has isomorphism type $\mu$ and the parabolic subgroup at $\infty$ is the standard one.

Specializing to one choice of parameters, we can consider changes of trivialization acting on $\mathrm{Gr}_{a_{1}, \ldots, a_{n}}$. Let $G_{1}\left(\mathbb{P}^{1} \backslash\left\{a_{1}, \ldots, a_{n}\right\}\right)$ denote the kernel of $G\left(\mathbb{P}^{1} \backslash\left\{a_{1}, \ldots, a_{n}\right\}\right) \rightarrow G$ given by evaluation at $\infty$. Then $\mathrm{Gr}_{\mu,\left(a_{1}, \ldots, a_{n}\right)}$ is an orbit of $G_{1}\left(\mathbb{P}^{1} \backslash\left\{a_{1}, \ldots, a_{n}\right\}\right)$.

We may also think of this locus in terms of the $\mathbb{C}^{\times}$action. We have an action of $\mathbb{C}^{\times}$on $\mathrm{Gr}_{\mathbb{A}^{n}}$ coming from the action of $\mathbb{C}^{\times}$on $\mathbb{P}^{1}$. Note that this action moves the base $\mathbb{A}^{n}$. On the central fiber $\operatorname{Gr}_{(0, \ldots, 0)}=\mathrm{Gr}$, this action of $\mathbb{C}^{\times}$restricts to the loop rotation action on $\mathrm{Gr}$. Hence the fixed points of this $\mathbb{C}^{\times}$action are the same as the fixed points of the loop rotation action, namely, the sets $G t^{\mu}$ inside the affine Grassmannian. Moreover, we have that $\mathrm{Gr}_{\mu, \mathbb{A}^{n}}$ is the attracting set for $t^{w_{0} \mu}$ under the $\mathbb{C}^{\times}$action. 
We have a fiberwise Poisson structure on $\mathrm{Gr}_{\mathbb{A}^{n}}$ using the Manin triples described in [Etingof and Kazhdan 1998, Corollary 2.10 and Proposition 2.12]. As in Section 2C, we get a Poisson structure on $\operatorname{Gr}_{\mu,\left(a_{1}, \ldots, a_{n}\right)}$.

Now let us choose an expression $\lambda=\lambda_{1}+\cdots+\lambda_{n}$, where $\lambda_{1}, \ldots, \lambda_{n}$ are fundamental coweights. This gives us a colored divisor $D$ on $\mathbb{P}^{1}$ defined by $D=$ $\sum \lambda_{i} a_{i}$. We will think of $D$ as a function on $\mathbb{P}^{1}$ with values in the dominant coweights. Now we define

$\operatorname{Gr}_{\mu,\left(a_{1}, \ldots, a_{n}\right)}^{\lambda_{1}, \ldots, \lambda_{n}}:=\left\{(E, \phi) \in \operatorname{Gr}_{\mu,\left(a_{1}, \ldots, a_{n}\right)} \mid(E, \phi)\right.$ has Hecke type $D(x)$ for all $\left.x \in \mathbb{P}^{1}\right\}$.

From the above analysis, it is possible to show that these are symplectic leaves in $\mathrm{Gr}_{\mu,\left(a_{1}, \ldots, a_{n}\right)}$.

Fixing $\left(\lambda_{1}, \ldots, \lambda_{n}\right)$ and letting $\left(a_{1}, \ldots, a_{n}\right)$ vary, this forms a family of $\mathbb{A}^{n}$. The central fiber of this family is $\mathrm{Gr}_{\mu}^{\lambda}$.

Now, define

$$
\begin{aligned}
& \operatorname{Gr}_{\mu,\left(a_{1}, \ldots, a_{n}\right)}^{\overline{\lambda_{1}, \ldots, \lambda_{n}}} \\
& \quad:=\left\{(E, \phi) \in \operatorname{Gr}_{\mu,\left(a_{1}, \ldots, a_{n}\right)} \mid(E, \phi) \text { has Hecke type } \leq D(x) \text { for all } x \in \mathbb{P}^{1}\right\} .
\end{aligned}
$$

Then we obtain a family of symplectic varieties over $\mathbb{A}^{n}$ whose central fiber is $\mathrm{Gr}_{\mu}^{\bar{\lambda}}$.

2E. Direct system on slices and Zastava spaces. We now look at what happens to $\mathrm{Gr}_{\mu}^{\bar{\lambda}}$ when we increase $\lambda, \mu$, keeping $\lambda-\mu$ fixed.

Let us fix $v$ in the positive coroot cone. Let $\mu, \mu^{\prime}$ be dominant coweights with $\mu^{\prime}-\mu$ dominant. From Lemma 2.3, we know that the stabilizer of $t^{w_{0} \mu^{\prime}}$ in $G_{1} \llbracket t^{-1} \rrbracket$ contains the stabilizer of $t^{w_{0} \mu}$ in $G_{1} \llbracket t^{-1} \rrbracket$. So we can define a map $\mathrm{Gr}_{\mu} \rightarrow \mathrm{Gr}_{\mu^{\prime}}$ by $g t^{w_{0} \mu} \mapsto g t^{w_{0} \mu^{\prime}}$. From Proposition 2.4, we see that this restricts to a map

$$
\mathrm{Gr}_{\mu}^{\overline{\mu+v}} \rightarrow \mathrm{Gr}_{\mu^{\prime}}^{\overline{\mu^{\prime}+v}}
$$

By construction, it is a Poisson map.

Clearly these maps are compatible with composition. Thus with $v$ fixed we get a direct system of slices $\left\{\mathrm{Gr}_{\mu}^{\overline{\mu+\nu}}\right\}_{\mu}$. The limit of this system is an ind-scheme, but in general it will not be represented by a scheme.

On the other hand, we can consider the Zastava space $Z_{v}$, an affine variety, as defined in [Finkelberg and Mirković 1999]. It is a partial compactification of the moduli space $Z_{v}^{\circ}$ of based maps from $\mathbb{P}^{1}$ into $G / B$ of degree $v$. The variety $Z_{v}$ carries an action of $\mathbb{C}^{\times}$, extending the action of $\mathbb{C}^{\times}$on $Z_{v}^{\circ}$ which rotates the source of the map.

The following result shows that the algebras of functions $O\left(\mathrm{Gr}_{\mu}^{\overline{\mu+\nu}}\right)$ stabilize to $O\left(Z_{v}\right)$.

Theorem 2.10 [Braverman and Finkelberg 2011, Theorem 2.8]. There exists a map $\mathrm{Gr}_{\mu}^{\overline{\mu+v}} \rightarrow Z_{v}$. These maps are compatible with the above direct system on the slices 
and with the actions of $\mathbb{C}^{\times}$. Moreover, the induced maps $\mathcal{O}\left(Z_{v}\right)_{N} \rightarrow \mathcal{O}\left(\mathrm{Gr}_{\mu}^{\overline{\mu+\nu}}\right)_{N}$ are isomorphisms if $N \leq\left\langle\alpha_{i}, \mu\right\rangle$ for all $i$.

Remark 2.11. The theorem provides $Z_{v}$ with a Poisson structure. On the other hand, $Z_{v}^{\circ}$ carries a symplectic structure as described in [Finkelberg et al. 1999]. It is expected that these two structures are compatible.

Example 2.12. Let us take $G=\mathrm{PGL}_{2}$ and $v=\alpha^{\vee}$, the simple coroot. Then (as in Example 2.2), for $n \geq 0$,

$$
\mathrm{Gr}_{n \omega^{\vee}}^{\overline{n \omega^{\vee}+\alpha^{\vee}}} \cong\left\{(u, v, w) \mid u v+w^{n+2}=0\right\} .
$$

Moreover, for $m \geq n$, the map $\operatorname{Gr}_{n \omega^{\vee}}^{\overline{n \omega^{\vee}+\alpha^{\vee}}} \rightarrow \operatorname{Gr}_{m \omega^{\vee}}^{\overline{m \omega^{\vee}+\alpha^{\vee}}}$ is given by $(u, v, w) \mapsto$ $\left(u, v w^{m-n}, w\right)$. This is because we have an equality in $\mathrm{Gr}_{\mathrm{PGL}_{2}}$ :

$$
\begin{aligned}
& {\left[\begin{array}{cc}
1-w t^{-1} & v t^{-(n+1)} \\
u t^{-1} & 1+w t^{-1}+\cdots+w^{n+1} t^{-(n+1)}
\end{array}\right]\left[\begin{array}{cc}
1 & 0 \\
0 & t^{m}
\end{array}\right]} \\
& =\left[\begin{array}{cc}
1-w t^{-1} & v w^{m-n} t^{-(m+1)} \\
u t^{-1} & 1+w t^{-1}+\cdots+w^{m+1} t^{-(m+1)}
\end{array}\right]\left[\begin{array}{cc}
1 & 0 \\
0 & t^{m}
\end{array}\right] .
\end{aligned}
$$

On the other hand, the Zastava space $Z_{\alpha}$ is $\mathbb{A}^{2}$. The map in Theorem 2.10 is given by $(u, v, w) \mapsto(u, w)$.

With respect to the $\mathbb{C}^{\times}$action on

$$
\operatorname{Gr}_{n \omega^{\vee}}^{\overline{n \omega^{\vee}+\alpha^{\vee}}}=\left\{(u, v, w) \mid u v+w^{n+2}=0\right\},
$$

the variables $u, w$ have weight 1 and $v$ has weight $n+1$. So we can see that

$$
\mathcal{O}\left(Z_{\alpha}\right)=\mathbb{C}[u, w] \rightarrow \mathcal{O}\left(\mathrm{Gr}_{n \omega^{\vee}}^{\overline{n \omega^{\vee}+\alpha^{\vee}}}\right)=\mathbb{C}[u, v, w] /\left(u v+w^{n+2}\right)
$$

is an isomorphism in degrees $0, \ldots, n$, as predicted by Theorem 2.10 .

The Poisson structure on $\mathrm{Gr}_{n \omega^{\vee}}^{n \omega^{\vee}+\alpha^{\vee}}$ is given by

$$
\{w, u\}=u, \quad\{w, v\}=-v, \quad\{u, v\}=(n+2) w^{n+1},
$$

while the Poisson structure on $Z_{\alpha}$ is given by

$$
\{w, u\}=u \text {. }
$$

Finally, note that the $\mathbb{C}$-points of the ind-scheme $\lim _{n} \mathrm{Gr}_{n \omega^{\vee}}^{\overline{n \omega^{\vee}+\alpha^{\vee}}}$ are

$$
\left\{(u, w) \mid u \in \mathbb{C}^{\times}, w \in \mathbb{C}\right\} \cup\{(0,0)\},
$$

which is a proper subset of $\mathbb{C}^{2}$, and hence this ind-scheme is not equal to $\mathbb{A}^{2}$. 
2F. Description of the Poisson structure. We would like to describe the Poisson structure on $G_{1} \llbracket t^{-1} \rrbracket$ in a little more detail. Let $C \in \mathfrak{g} \otimes \mathfrak{g}$ be the Casimir element for the bilinear form. Picking dual bases, we may represent this element as $C=\sum J_{a} \otimes J^{a}$; this Casimir element allows us to describe the Poisson bracket of two minors. This can be written more compactly using the series

$$
\Delta_{\beta, v}(u)=\sum_{s \geq 0} \Delta_{\beta, v}^{(s)} u^{-s}
$$

Note that $\Delta_{\beta, v}^{(0)}=\langle\beta, w\rangle$ is a constant function.

Proposition 2.13. The Poisson bracket $\left\{\Delta_{\beta_{1}, v_{1}}\left(u_{1}\right), \Delta_{\beta_{2}, v_{2}}\left(u_{2}\right)\right\}$ is equal to

$$
\frac{1}{u_{1}-u_{2}} \sum_{a} \Delta_{\beta_{1}, J_{a} v_{1}}\left(u_{1}\right) \Delta_{\beta_{2}, J^{a} v_{2}}\left(u_{2}\right)-\Delta_{J_{a} \beta_{1}, v_{1}}\left(u_{1}\right) \Delta_{J^{a} \beta_{2}, v_{2}}\left(u_{2}\right)
$$

in $O\left(G_{1} \llbracket t^{-1} \rrbracket\right) \llbracket u_{1}^{-1}, u_{2}^{-1} \rrbracket$.

Proof. The cobracket $\mathfrak{g}\left(\left(t^{-1}\right)\right) \rightarrow \mathfrak{g}\left(\left(u_{1}\right)\right) \otimes \mathfrak{g}\left(\left(u_{2}\right)\right)$ is coboundary. If we let $r\left(u_{1}, u_{2}\right)=C /\left(u_{1}-u_{2}\right)$, it is given by

$$
a(t) \mapsto\left[a\left(u_{1}\right) \otimes 1+1 \otimes a\left(u_{2}\right), r\left(u_{1}, u_{2}\right)\right] .
$$

As described earlier, the Lie algebra $\mathfrak{g}\left(\left(t^{-1}\right)\right)$ carries an inner product

$$
(f, g)_{t}=-\operatorname{res}_{t=0}(f, g)
$$

for which $t^{-1} \mathfrak{g} \llbracket t^{-1} \rrbracket$ is Lagrangian and complementary to $\mathfrak{g}[t]$; this realizes $\mathfrak{g}\left(\left(t^{-1}\right)\right)$ as the (topological) Drinfeld double of $t^{-1} \mathfrak{g} \llbracket t^{-1} \rrbracket$. In particular,

$$
G_{1} \llbracket t^{-1} \rrbracket \subset G\left(\left(t^{-1}\right)\right)
$$

is a Poisson subgroup, and the Poisson bracket of any two functions on $G_{1} \llbracket t^{-1} \rrbracket$ can be calculated taking the bracket of any two extensions to all of $G\left(\left(t^{-1}\right)\right)$ and then restricting to $G_{1} \llbracket t^{-1} \rrbracket$.

Thus, the Poisson structure on $G\left(\left(t^{-1}\right)\right)$ is defined by

$$
\pi=r^{L}\left(u_{1}, u_{2}\right)-r^{R}\left(u_{1}, u_{2}\right),
$$

the difference of the left translation and right translation of the element $r\left(u_{1}, u_{2}\right)$ considered as a bivector at the identity. If $X \in t^{-1} \mathfrak{g} \llbracket t^{-1} \rrbracket$ and $g \in G_{1} \llbracket t^{-1} \rrbracket$, we identify $X$ with a tangent vector at $g$ by left translation. Then we have

$$
\left(d \Delta_{\beta, v}\right)_{g}(X)=\langle\beta, g X v\rangle .
$$


Hence

$$
\begin{aligned}
& \left\{\Delta_{\beta_{1}, v_{1}}\left(u_{1}\right), \Delta_{\beta_{2}, v_{2}}\left(u_{2}\right)\right\}(g) \\
& =\left\langle\pi,\left(d \Delta_{\beta_{1}, v_{1}}\right)_{g} \otimes\left(d \Delta_{\beta_{2}, v_{2}}\right)_{g}(g)\right\rangle \\
& =\left\langle r^{L}\left(u_{1}, u_{2}\right)-r^{R}\left(u_{1}, u_{2}\right),\left(d \Delta_{\beta_{1}, v_{1}}\right)_{g} \otimes\left(d \Delta_{\beta_{2}, v_{2}}\right)_{g}(g)\right\rangle \\
& =\frac{1}{u_{1}-u_{2}}\left(\sum_{a}\left\langle\beta_{1}, g\left(u_{1}\right) J_{a} v_{1}\right\rangle\left\langle\beta_{2}, g\left(u_{2}\right) J^{a} v_{2}\right\rangle\right.
\end{aligned}
$$

where the last step follows from the invariance of the pairing between dual representations.

We can unpack Proposition 2.13 into the following equations:

$$
\left\{\Delta_{\beta_{1}, v_{1}}^{(r+1)}, \Delta_{\beta_{2}, v_{2}}^{(s)}\right\}-\left\{\Delta_{\beta_{1}, v_{1}}^{(r)}, \Delta_{\beta_{2}, v_{2}}^{(s+1)}\right\}=\sum \Delta_{J_{a} \beta_{1}, v_{1}}^{(r)} \Delta_{J^{a} \beta_{2}, v_{2}}^{(s)}-\Delta_{\beta_{1}, J_{a} v_{1}}^{(r)} \Delta_{\beta_{2}, J^{a} v_{2}}^{(s)}
$$

for $r, s \geq 0$. These equations specify all the desired Poisson brackets.

2G. A conjectural description of the ideal of $\mathrm{Gr}_{\mu}^{\bar{\lambda}}$. In this section, we give a conjectural description of the ideal of $\mathrm{Gr}_{\mu}^{\bar{\lambda}}$ as a subvariety of $\mathrm{Gr}_{0}=G_{1} \llbracket t^{-1} \rrbracket$. Let $G^{\text {sc }}$ denote the simply connected cover of $G$. Note that the natural map $G_{1}^{\mathrm{sc}} \llbracket t^{-1} \rrbracket \rightarrow G_{1} \llbracket t^{-1} \rrbracket$ is an isomorphism. This allows us to consider $\Delta_{\omega_{i}, \omega_{i}}^{(s)}$ as functions on $G_{1} \llbracket t^{-1} \rrbracket$, even if $\omega_{i}$ are not weights of $G$ (for example if $G$ is of adjoint type).

We begin with the case of $\mu=0$. Let $J_{0}^{\lambda}$ denote the ideal in $\mathcal{O}\left(G_{1} \llbracket t^{-1} \rrbracket\right)$ Poisson generated by $\Delta_{\omega_{i}, \omega_{i}}^{(s)}$ for $\left.s\right\rangle\left\langle\lambda, \omega_{i *}\right\rangle$ and for $i \in I$.

Conjecture 2.14. The ideal of $\mathrm{Gr}_{0}^{\bar{\lambda}}$ in $\mathrm{O}\left(G_{1} \llbracket t^{-1} \rrbracket\right)$ is $J_{0}^{\lambda}$.

Let us make some comments on this conjecture. First, we have the following result.

Proposition 2.15. $J_{0}^{\lambda}$ is generated as an ordinary ideal by $\Delta_{\beta, v}^{(s)}$ for $\left.s\right\rangle\left\langle\lambda, \omega_{i^{*}}\right\rangle$ and for $i \in I$, where $\beta, v$ range over bases for $V\left(\omega_{i}\right)^{*}$ and $V\left(\omega_{i}\right)$.

Proof. Let $I$ be the ideal generated as an ordinary ideal by $\Delta_{\beta, v}^{(s)}$ for $s>\left\langle\lambda, \omega_{i^{*}}\right\rangle$. First, we show that this ideal is contained in $J_{0}^{\lambda}$.

We claim that $\Delta_{\omega_{i}, v}^{(s)} \in J_{0}^{\lambda}$ for all $v \in V\left(\omega_{i}\right)$ and $s>\left\langle\lambda, \omega_{i *}\right\rangle$. We proceed by downward induction on the weight of $v$. The base case of $v$ is highest weight 
follows by definition. For the inductive step, suppose that $v$ is not highest weight. In this case, $v=\sum f_{j} v_{j}$ for some $v_{j}$ of higher weight than $v$.

Fix $s$ with $s\rangle\left\langle\lambda, \omega_{i^{*}}\right\rangle$. Using (1) with $s=0$ and the expression for the Casimir (for notation see Section 3B)

$$
C=C_{\mathfrak{h}}+\sum_{\alpha \in \Phi_{+}} C_{\alpha} e_{\alpha} \otimes f_{\alpha}+C_{\alpha} f_{\alpha} \otimes e_{\alpha}
$$

where $\left(e_{\alpha}, f_{\alpha}\right)=C_{\alpha}^{-1}$, we see that

$$
\left\{\Delta_{\omega_{i}, v_{j}}^{(r)}, \Delta_{\omega_{j}, s_{j} \omega_{j}}^{(1)}\right\}=-\Delta_{\omega_{i}, f_{j} v_{j}}^{(r)} .
$$

Thus we see that

$$
\Delta_{\omega_{i}, v}^{(s)}=\sum_{j} \Delta_{\omega_{i}, f_{j} v_{j}}^{(s)}=-\sum_{j}\left\{\Delta_{\omega_{i}, v_{j}}^{(s)}, \Delta_{\omega_{j}, s_{j} \omega_{j}}^{(1)}\right\}
$$

All the terms on the right-hand side lie in $J_{0}^{\lambda}$ by the inductive assumption, and thus $\Delta_{\omega_{i}, v}^{(s)} \in J_{0}^{\lambda}$.

Now we claim that $\Delta_{\beta, v}^{(s)} \in J_{0}^{\lambda}$ for all $\beta \in V\left(\omega_{i}\right)^{*}, v \in V\left(\omega_{i}\right)$, and $s>\left\langle\lambda, \omega_{i^{*}}\right\rangle$.

We have already proven this claim when $\beta=v_{-\omega_{i}}$, so we proceed by induction on the weight of $\beta$. Suppose that $\beta \in V\left(\omega_{i}\right)^{*}$ is not lowest weight and assume that the claim holds for all $\beta$ of lower weight. In this case, we can write $\beta=\sum e_{j} \beta_{j}$ for some $\beta_{j}$ of lower weight.

Fix $s$ with $s\rangle\left\langle\lambda, \omega_{i^{*}}\right\rangle$. Again using the above expression for the Casimir, we find that

$$
\left\{\Delta_{\beta_{j}, v}^{(s)}, \Delta_{s_{j} \omega_{j}, \omega_{j}}^{(1)}\right\}=\Delta_{\beta_{j}, e_{j} v}^{(s)}-\Delta_{e_{j} \beta_{j}, v}^{(s)} .
$$

Thus we see that

$$
\Delta_{\beta, v}^{(s)}=\sum_{j} \Delta_{e_{j} \beta_{j}, v}^{(s)}=\sum_{j}\left\{\Delta_{\beta_{j}, v}^{(s)}, \Delta_{s_{j} \omega_{j}, \omega_{j}}^{(1)}\right\}-\Delta_{\beta_{j}, e_{j} v}^{(s)} .
$$

All the terms on the right-hand side lie in $J_{0}^{\lambda}$ by the inductive assumption, and thus $\Delta_{\beta, v}^{(s)} \in J_{0}^{\lambda}$. This shows that $I \subset J_{\lambda}^{0}$.

It remains to show that $I$ is a Poisson ideal. Since $\Delta_{\beta, v}^{(s)}$, for $\beta \in V\left(\omega_{i}\right)^{*}, v \in$ $V\left(\omega_{i}\right), i \in I$, generates $\mathcal{O}\left(G_{1} \llbracket t^{-1} \rrbracket\right)$, it suffices to check that $I$ is closed under Poisson bracket with these elements. This follows immediately from (1).

Combining this proposition with Proposition 2.4, we obtain the following.

Corollary 2.16. The vanishing set of $J_{0}^{\lambda}$ is $\mathrm{Gr}_{0}^{\bar{\lambda}}$.

Thus in order to establish Conjecture 2.14, it only remains to show that $I_{0}^{\lambda}$ is radical. 
Remark 2.17. Let $G=\mathrm{SL}_{n}$. By an observation which goes back to Lusztig [1981, Section 2], we know that there is an isomorphism $\mathrm{Gr}_{0}^{\frac{g \omega_{1}}{n}} \cong \mathcal{N}$, the nilpotent cone of $\mathfrak{s l}_{n}$. For any dominant coweight $\lambda$ with $\lambda \leq n \omega_{1}$, under this isomorphism $\mathrm{Gr}_{0}^{\bar{\lambda}}$ is taken to a nilpotent orbit closure. Thus, the above conjecture implies generators for the ideal of a nilpotent orbit closure inside the nilpotent cone of $\mathfrak{s l}_{n}$. From this perspective, one can see that Conjecture 2.14 would imply the main result of [Weyman 1989], which gives generators for the ideals of nilpotent orbit closures. This gives additional evidence toward the conjecture, but also suggests it will be difficult to prove.

Remark 2.18. One could imagine a similar conjecture for the ideal of $\mathrm{Gr}^{\bar{\lambda}}$ inside of the homogeneous coordinate ring of $\mathrm{Gr}$ with respect to its natural determinant line bundle. However, this conjecture is false, already for $\mathrm{SL}_{2}$ and $\lambda=\alpha$.

We will need the following generalization of Conjecture 2.14, which describes the ideal of $\mathrm{Gr}_{\mu}^{\bar{\lambda}}$. Consider the subgroup $G_{1} \llbracket t^{-1} \rrbracket_{\mu}$ defined as the stabilizer in $G_{1} \llbracket t^{-1} \rrbracket$ of $t^{w_{0} \mu}$. Note that, by Lemma 2.3, $G_{1} \llbracket t^{-1} \rrbracket_{\mu} \subset N_{1} \llbracket t^{-1} \rrbracket$.

By the orbit-stabilizer theorem, we see that $\mathrm{Gr}_{\mu}=G_{1} \llbracket t^{-1} \rrbracket / G_{1} \llbracket t^{-1} \rrbracket_{\mu}$, and so $\left.\mathcal{O}\left(\mathrm{Gr}_{\mu}\right)=\mathcal{O}\left(G_{1} \llbracket t^{-1} \rrbracket\right)\right)^{G_{1} \llbracket t^{-1} \rrbracket_{\mu}}$. Moreover, the map $G_{1} \llbracket t^{-1} \rrbracket \rightarrow \mathrm{Gr}_{\mu}$ is Poisson, and thus $\mathcal{O}\left(\mathrm{Gr}_{\mu}\right)$ is a Poisson subalgebra of $\mathrm{O}\left(G_{1} \llbracket t^{-1} \rrbracket\right)$.

Lemma 2.19. The subalgebra $\mathrm{O}\left(\mathrm{Gr}_{\mu}\right)$ contains

$$
\begin{aligned}
\Delta_{s_{i} \omega_{i}, \omega_{i}}^{(s)} & \text { for all } i \in I, s>0, \\
\Delta_{\omega_{i}, \omega_{i}}^{(s)} & \text { for all } i \in I, s>0, \\
\left(\Delta_{\omega_{i}, s_{i} \omega_{i}} / \Delta_{\omega_{i}, \omega_{i}}\right)^{(s)} & \text { for all } i \in I, s>\left\langle\mu^{*}, \alpha_{i}\right\rangle .
\end{aligned}
$$

Later we will see that these elements generate $\mathcal{O}\left(\mathrm{Gr}_{\mu}\right)$ as a Poisson algebra.

Proof. Note that the action of $G_{1} \llbracket t^{-1} \rrbracket_{\mu}$ on $\mathcal{O}\left(G_{1} \llbracket t^{-1} \rrbracket\right)$ is given by $(k \cdot f)(g)=$ $g(f k)$ for $k \in G_{1} \llbracket t^{-1} \rrbracket_{\mu}, f \in \mathcal{O}\left(G_{1} \llbracket t^{-1} \rrbracket\right)$, and $g \in G_{1} \llbracket t^{-1} \rrbracket$. In particular, we see that $k \cdot \Delta_{\beta, v}=\Delta_{\beta, k v}$.

Since $G_{1} \llbracket t^{-1} \rrbracket_{\mu} \subset N_{1} \llbracket t^{-1} \rrbracket$, the minors $\Delta_{\omega_{i}, \omega_{i}}$ and $\Delta_{s_{i} \omega_{i}, \omega_{i}}$ will be $G_{1} \llbracket t^{-1} \rrbracket_{\mu^{-}}$ invariant. Hence all $\Delta_{s_{i} \omega_{i}, \omega_{i}}^{(s)}, \Delta_{\omega_{i}, \omega_{i}}^{(s)}$ lie in $\mathcal{O}\left(\mathrm{Gr}_{\mu}\right)$.

On the other hand, let us consider the coefficients of the $\Delta_{\omega_{i}, s_{i} \omega_{i}}$ minor. If $k \in G_{1} \llbracket t^{-1} \rrbracket_{\mu}$, then we have $k \cdot v_{s_{i} \omega_{i}}=v_{s_{i} \omega_{i}}+\Delta_{\omega_{i}, s_{i} \omega_{i}}(k) v_{\omega_{i}}$. Hence if $g \in G_{1} \llbracket t^{-1} \rrbracket$, then

$$
\frac{\Delta_{\omega_{i}, s_{i} \omega_{i}}(g k)}{\Delta_{\omega_{i}, \omega_{i}}(g k)}=\frac{\Delta_{\omega_{i}, s_{i} \omega_{i}}(g)+\Delta_{\omega_{i}, \omega_{i}}(g) \Delta_{\omega_{i}, s_{i} \omega_{i}}(k)}{\Delta_{\omega_{i}, \omega_{i}}(g)}=\frac{\Delta_{\omega_{i}, s_{i} \omega_{i}}(g)}{\Delta_{\omega_{i}, \omega_{i}}(g)}+\Delta_{\omega_{i}, s_{i} \omega_{i}}(k) .
$$

By Lemma 2.3, we have $\Delta_{\omega_{i}, s_{i} \omega_{i}}(k) \in t^{-\left\langle w_{0} \mu, \alpha_{i}\right\rangle} \mathbb{C}[t]$. Hence the coefficient of $t^{-s}$ in $\Delta_{\omega_{i}, s_{i} \omega_{i}} / \Delta_{\omega_{i}, \omega_{i}}$ is invariant under the action of $G_{1} \llbracket t^{-1} \rrbracket_{\mu}$ for $s>\left\langle\mu^{*}, \alpha_{i}\right\rangle$. Thus $\left(\Delta_{\omega_{i}, s_{i} \omega_{i}} / \Delta_{\omega_{i}, \omega_{i}}\right)^{(s)} \in \mathcal{O}\left(\mathrm{Gr}_{\mu}\right)$ for $s>\left\langle\mu^{*}, \alpha_{i}\right\rangle$. 
Let $J_{\mu}^{\lambda}$ denote the ideal of $\mathcal{O}\left(\mathrm{Gr}_{\mu}\right)$ Poisson generated by $\Delta_{\omega_{i}, \omega_{i}}^{(s)}$ for $i \in I$ and $s>\left\langle\lambda-\mu, \omega_{i *}\right\rangle=m_{i}$.

Conjecture 2.20. The ideal of $\mathrm{Gr}_{\mu}^{\bar{\lambda}}$ in $\mathrm{O}\left(\mathrm{Gr}_{\mu}\right)$ is $J_{\mu}^{\lambda}$.

This conjecture generalizes Conjecture 2.14. When $\mu \neq 0$, we do not have a set of (ordinary) generators for $J_{\mu}^{\lambda}$ as in Proposition 2.15. However, we will now establish an analogue of Corollary 2.16.

Proposition 2.21. The vanishing locus of $J_{\mu}^{\lambda}$ is $\mathrm{Gr}_{\mu}^{\bar{\lambda}}$.

Proof. The vanishing locus of $J_{\mu}^{\lambda}$ is the union of the symplectic leaves in the vanishing locus of $\Delta_{\omega_{i}}^{(s)}, \omega_{i}$ for $i \in I$ and $\left.s\right\rangle\left\langle\lambda-\mu, \omega_{i^{*}}\right\rangle=m_{i}$; after all, the vanishing set is a union of symplectic leaves and if these functions vanish on a symplectic leaf, then so do all Poisson brackets with them.

These generalized minors vanish on $\mathrm{Gr}_{\mu}^{\bar{\lambda}}$ by Proposition 2.4. So it suffices to prove the vanishing locus of our generators does not contain $\mathrm{Gr}_{\mu}^{v}$ for some $v \not \lambda$.

Fix $v \not \leq \lambda$ such that $\mu \leq v$. Then $\left.d=\left\langle\nu-\mu, \omega_{i^{*}}\right\rangle\right\rangle\left\langle\lambda-\mu, \omega_{i^{*}}\right\rangle$ for some $i$. We will prove that there exists a point in $\operatorname{Gr}_{\mu}^{v}$ on which $\Delta_{\omega_{i}, \omega_{i}}^{(d)}$ is nonzero.

Let $I_{+}^{+}=I \subset G\left(\left(t^{-1}\right)\right)$ denote the standard Iwahori and let $I_{-}^{+}=w_{0} I_{+}^{+} w_{0}^{-1}$ be the preimage of $B_{-}$in $G[t]$. We claim that it suffices to prove that

$$
I_{-}^{+} t^{w_{0} v} I_{+}^{+} \cap G_{-} \llbracket t^{-1} \rrbracket t^{w_{0} \mu} \neq \varnothing \quad \text { in } G\left(\left(t^{-1}\right)\right) .
$$

To see that (2) suffices, let $g \in G_{1} \llbracket t^{-1} \rrbracket$ such that $g t^{w_{0} \mu}$ lies in the above intersection. As $I_{-}^{+}, I_{+}^{+} \subset G[t]$, we see that $g t^{w_{0} \mu} \in \mathrm{Gr}_{\mu}^{v}$. Finally, we can write $g=b_{-} t^{w_{0} v} b_{+} t^{-w_{0} \mu}$ for $b_{-} \in I_{-}^{+}, b_{+} \in I_{+}^{+}$, and an elementary computation shows that $\Delta_{\omega_{i}, \omega_{i}}^{(d)}\left(b_{-} t^{w_{0} v} b_{+} t^{-w_{0} \mu}\right) \neq 0$.

To prove (2), we work in the affine flag variety $G\left(\left(t^{-1}\right)\right) / I$ and note that (2) is equivalent to nonemptiness of the intersection $I_{-}^{+} t^{w_{0} v} \cap G_{-} \llbracket t^{-1} \rrbracket t^{w_{0} \mu}$ in $G\left(\left(t^{-1}\right)\right) / I$. Let $I_{+}^{-}$denote the preimage of $B$ in $G \llbracket t^{-1} \rrbracket$ under evaluation at $t^{-1}=0$. Since $\mu$ is dominant, $B$ fixes $t^{w_{0} \mu}$ and thus $G_{-} \llbracket t^{-1} \rrbracket t^{w_{0} \mu}=I_{+}^{-} t^{w_{0} \mu}$. Thus we reduce to proving that

$$
I_{-}^{+} t^{w_{0} v} \cap I_{+}^{-} t^{w_{0} \mu} \neq \varnothing \quad \text { in } G\left(\left(t^{-1}\right)\right) / I
$$

Twisting by $w_{0}$, we reduce to proving that

$$
I_{+}^{+} w_{0} t^{w_{0} v} \cap I_{-}^{-} w_{0} t^{w_{0} \mu} \neq \varnothing \quad \text { in } G\left(\left(t^{-1}\right)\right) / I
$$

where $I_{-}^{-}$is the preimage of $B_{-}$in $G \llbracket t^{-1} \rrbracket$. From general theory of flag varieties, this is equivalent to $w_{0} t^{w_{0} v} \geq w_{0} t^{w_{0} \mu}$ in the Bruhat order on the (extended) affine Weyl group. This last fact is easily verified under our hypothesis that $\mu, v$ are dominant and $v \geq \mu$. 


\section{Yangians}

3A. The Drinfeld Yangian. As mentioned in the introduction, we will study subquotients of Yangians in order to quantize our slices. We will actually need a slight variant on the usual Yangian, which will be produced via a theory developed by Gavarini [2007; 2002]. We begin with the usual Yangian, which we call the Drinfeld Yangian to avoid confusion with the Yangian we wish to consider.

We define the Drinfeld Yangian $U_{h} \mathfrak{g}[t]$ as the associative $\mathbb{C} \llbracket h \rrbracket$-algebra with generators $e_{i}^{(s)}, h_{i}^{(s)}, f_{i}^{(s)}$ for $i \in I$ and $r, s \in \mathbb{N}$ and relations

$$
\begin{aligned}
{\left[h_{i}^{(s)}, h_{j}^{(s)}\right] } & =0, \\
{\left[e_{i}^{(r)}, f_{i}^{(s)}\right] } & =\delta_{i j} h_{i}^{(r+s)}, \\
{\left[h_{i}^{(0)}, e_{j}^{(s)}\right] } & =\left(\alpha_{i}, \alpha_{j}\right) e_{j}^{(s)}, \\
{\left[h_{i}^{(r+1)}, e_{j}^{(s)}\right]-\left[h_{i}^{(r)}, e_{j}^{(s+1)}\right] } & =\frac{h\left(\alpha_{i}, \alpha_{j}\right)}{2}\left(h_{i}^{(r)} e_{j}^{(s)}+e_{j}^{(s)} h_{i}^{(r)}\right), \\
{\left[h_{i}^{(0)}, f_{j}^{(s)}\right] } & =-\left(\alpha_{i}, \alpha_{j}\right) f_{j}^{(s)}, \\
{\left[h_{i}^{(r+1)}, f_{j}^{(s)}\right]-\left[h_{i}^{(r)}, f_{j}^{(s+1)}\right] } & =-\frac{h\left(\alpha_{i}, \alpha_{j}\right)}{2}\left(h_{i}^{(r)} f_{j}^{(s)}+f_{j}^{(s)} h_{i}^{(r)}\right), \\
{\left[e_{i}^{(r+1)}, e_{j}^{(s)}\right]-\left[e_{i}^{(r)}, e_{j}^{(s+1)}\right] } & =\frac{h\left(\alpha_{i}, \alpha_{j}\right)}{2}\left(e_{i}^{(r)} e_{j}^{(s)}+e_{j}^{(s)} e_{i}^{(r)}\right), \\
{\left[f_{i}^{(r+1)}, f_{j}^{(s)}\right]-\left[f_{i}^{(r)}, f_{j}^{(s+1)}\right] } & =-\frac{h\left(\alpha_{i}, \alpha_{j}\right)}{2}\left(f_{i}^{(r)} f_{j}^{(s)}+f_{j}^{(s)} f_{i}^{(r)}\right), \\
i \neq j, N=1-a_{i j} & \Rightarrow \operatorname{sym}\left[e_{i}^{\left(r_{1}\right)},\left[e_{i}^{\left(r_{2}\right)}, \ldots\left[e_{i}^{\left(r_{N}\right)}, e_{j}^{(s)}\right] \ldots\right]\right]=0, \\
i \neq j, N=1-a_{i j} & \Rightarrow \operatorname{sym}\left[f_{i}^{\left(r_{1}\right)},\left[f_{i}^{\left(r_{2}\right)}, \ldots\left[f_{i}^{\left(r_{N}\right)}, f_{j}^{(s)}\right] \ldots\right]\right]=0,
\end{aligned}
$$

where sym denotes symmetrization with respect to $r_{1}, \ldots, r_{N}$.

The following result of Drinfeld [1987, Example 6.3] will be our starting point.

Theorem 3.1. $U_{h} \mathfrak{g}[t]$ is a quantization of $\mathfrak{g}[t]$. More precisely, there is an isomorphism of co-Poisson Hopf algebras $U_{h} \mathfrak{g}[t] / h U_{h} \mathfrak{g}[t] \cong U \mathfrak{g}[t]$, where $U \mathfrak{g}[t]$ carries the co-Poisson structure coming from the Manin triple $\left(\mathfrak{g}[t], t^{-1} \mathfrak{g} \llbracket t^{-1} \rrbracket, \mathfrak{g}\left(\left(t^{-1}\right)\right)\right)$.

3B. PBW basis for the Drinfeld Yangian. Fix any order on the nodes of the Dynkin diagram; for each positive root $\alpha$, we let $\check{\alpha}$ denote the smallest simple root such that $\hat{\alpha}=\alpha-\check{\alpha}$ is again a positive root.

We define $e_{\alpha} \in \mathfrak{g}$ for $\alpha \in \Delta_{+}$recursively by

$$
e_{\alpha_{i}}=e_{i} \quad \text { and } \quad e_{\alpha}=\left[e_{\hat{\alpha}}, e_{\check{\alpha}}\right] .
$$

We extend this definition to $U_{h} \mathfrak{g}[t]$ by setting 


$$
e_{\alpha_{i}}^{(r)}=e_{i}^{(r)} \quad \text { and } \quad e_{\alpha}^{(r)}=\left[e_{\hat{\alpha}}^{(r)}, e_{\check{\alpha}}^{(0)}\right] .
$$

Similarly, we define $f_{\alpha}$ and $f_{\alpha}^{(r)}$. We have the following PBW theorem for the Drinfeld Yangian due to Levendorskii [1993].

Proposition 3.2. (1) Under the isomorphism $U_{h} \mathfrak{g}[t] / h U_{h} \mathfrak{g}[t] \cong U \mathfrak{g}[t]$, $e_{\alpha}^{(r)}$ corresponds to $e_{\alpha} t^{r}$.

(2) Ordered monomials in the $e_{\alpha}^{(r)}, h_{i}^{(r)}, f_{\beta}^{(r)}$ form a PBW basis for $U_{h} \mathfrak{g}[t]$.

3C. Drinfeld-Gavarini duality. Our goal is to give a quantization of the PoissonHopf algebra $\mathcal{O}\left(G_{1} \llbracket t^{-1} \rrbracket\right)$ using the Drinfeld Yangian $U_{h} \mathfrak{g}[t]$. For this we will use the quantum groups duality of Drinfeld and Gavarini.

We describe in brief one half of Drinfeld-Gavarini duality [Drinfeld 1987; Gavarini 2007; 2002]. Let $(H, \Delta, \epsilon)$ be a Hopf algebra over $\mathbb{C} \llbracket h \rrbracket$. Consider maps $\Delta^{n}: H \rightarrow H^{\otimes n}$ for $n \geq 0$ defined by $\Delta^{0}=\epsilon, \Delta^{1}=\mathrm{id}_{H}$, and

$$
\Delta^{n}=\left(\Delta \otimes \mathrm{id}^{\otimes(n-2)}\right) \circ \Delta^{n-1}
$$

for $n \geq 2$. Let $\delta^{n}=\left(\mathrm{id}_{H}-\epsilon\right)^{\otimes n} \circ \Delta^{n}$, and define the Hopf subalgebra

$$
H^{\prime}=\left\{a \in H \mid \delta^{n}(a) \in h^{n} H^{\otimes n}\right\} .
$$

In general, $H^{\prime} / h H^{\prime}$ is a commutative Hopf algebra over $\mathbb{C}$ and can be given the Poisson bracket

$$
\left\{a+h H^{\prime}, b+h H^{\prime}\right\}=h^{-1}[a, b]+h H^{\prime} .
$$

Suppose that $G$ is a Poisson affine algebraic group, namely the maximal spectrum of a Poisson commutative Hopf algebra $\mathcal{O}(G)$, and let $\mathfrak{g}, \mathfrak{g}^{*}$ be its tangent and cotangent Lie bialgebras. Let $U_{h}=U_{h}(\mathfrak{g})$ be a quantization of $U(\mathfrak{g})$.

Theorem 3.3 [Gavarini 2007, Theorem 2.2]. There is an isomorphism of PoissonHopf algebras

$$
U_{h}{ }^{\prime} / h U_{h}{ }^{\prime} \cong \mathcal{O}\left(G^{*}\right),
$$

where $G^{*}$ is a connected algebraic group with tangent Lie bialgebra $\mathfrak{g}^{*}$.

By [Gavarini 2002], for any basis $\left\{\bar{x}_{\alpha}\right\}$ of $\mathfrak{g}$, there exists a lift $\left\{x_{\alpha}\right\}$ in $U_{h}$ such that

- $\epsilon\left(x_{\alpha}\right)=0$,

- $U_{h}{ }^{\prime}$ is generated by $\left\{h x_{\alpha}\right\}$, and

- ordered monomials in these generators span $U_{h}{ }^{\prime}$ over $\mathbb{C} \llbracket h \rrbracket$.

In particular, if $\left\{\bar{x}_{i}\right\}$ generates $\mathfrak{g}$, then $\left\{h x_{i}+h U_{h}{ }^{\prime}\right\}$ generates $U_{h}{ }^{\prime} / h U_{h}{ }^{\prime}$ as a Poisson algebra. 
To allow for easier identification of $U_{h}{ }^{\prime} / h U_{h}{ }^{\prime}$ and $\mathcal{O}\left(G^{*}\right)$, we can reformulate Theorem 3.3 as follows. Consider

$$
\mathscr{L}=\operatorname{Der}\left(U_{h}{ }^{\prime} / h U_{h}{ }^{\prime}\right):=\left\{\varphi: U_{h}{ }^{\prime} / h U_{h}{ }^{\prime} \rightarrow \mathbb{C} \mid \varphi(a b)=\varphi(a) \epsilon(b)+\epsilon(a) \varphi(b)\right\},
$$

with Lie bracket

$$
[\varphi, \phi](a)=(\varphi \otimes \phi)\left(\Delta(a)-\Delta^{\mathrm{op}}(a)\right)
$$

and cobracket

$$
\delta(\varphi)(a \otimes b)=\varphi(\{a, b\}) .
$$

This is the Lie bialgebra of the Poisson algebraic group $\operatorname{Spec}\left(U_{h}{ }^{\prime} / h U_{h}{ }^{\prime}\right)$.

The isomorphism described in Theorem 3.3 can be rephrased as follows.

Corollary 3.4. There is an isomorphism of Lie bialgebras $\mathfrak{g}^{*} \cong \mathscr{L}$ defined by

$$
\bar{y} \mapsto\left(h x+h U_{h}{ }^{\prime} \mapsto\langle\bar{y}, \bar{x}\rangle\right)
$$

for $x$ a lift of $\bar{x} \in \mathfrak{g}$, extended by the Leibniz rule. This isomorphism yields a perfect Poisson-Hopf pairing $\langle\cdot, \cdot\rangle: U\left(\mathfrak{g}^{*}\right) \times U_{h}{ }^{\prime} / h U_{h}{ }^{\prime} \rightarrow \mathbb{C}$.

3D. Our Yangian. We will now apply this theory to the Drinfeld Yangian $U_{h} \mathfrak{g}[t]$. We let $Y:=\left(U_{h} \mathfrak{g}[t]\right)^{\prime}$. We will refer to $Y$ as the Yangian from now on. Note that it is a subalgebra of the usual Yangian.

For $X=E_{\alpha}, H_{i}, F_{\alpha}$ and $r \geq 1$, we define $X^{(r)}=h x^{(r-1)}$. By the general remarks above, these elements generate $Y$ and monomials in these generators give a PBW basis for $Y$. We define a grading on $Y$ where $X^{(r)}$ has degree $r$.

Theorem 3.5. The $X^{(r)}$ generate $Y$ subject to the relations

$$
\begin{aligned}
{\left[H_{i}^{(s)}, H_{j}^{(s)}\right] } & =0, \\
{\left[E_{i}^{(r)}, F_{j}^{(s)}\right] } & =h \delta_{i j} H_{i}^{(r+s-1)}, \\
{\left[H_{i}^{(1)}, E_{j}^{(s)}\right] } & =h\left(\alpha_{i}, \alpha_{j}\right) E_{j}^{(s)}, \\
{\left[H_{i}^{(r+1)}, E_{j}^{(s)}\right]-\left[H_{i}^{(r)}, E_{j}^{(s+1)}\right] } & =\frac{1}{2} h\left(\alpha_{i}, \alpha_{j}\right)\left(H_{i}^{(r)} E_{j}^{(s)}+E_{j}^{(s)} H_{i}^{(r)}\right), \\
{\left[H_{j}^{(1)}, F_{j}^{(s)}\right] } & =-h\left(\alpha_{i}, \alpha_{j}\right) F_{j}^{(s)}, \\
{\left[H_{i}^{(r+1)}, F_{j}^{(s)}\right]-\left[H_{i}^{(r)}, F_{j}^{(s+1)}\right] } & =-\frac{1}{2} h\left(\alpha_{i}, \alpha_{j}\right)\left(H_{i}^{(r)} F_{j}^{(s)}+F_{j}^{(s)} H_{i}^{(r)}\right), \\
{\left[E_{i}^{(r+1)}, E_{j}^{(s)}\right]-\left[E_{i}^{(r)}, E_{j}^{(s+1)}\right] } & =\frac{1}{2} h\left(\alpha_{i}, \alpha_{j}\right)\left(E_{i}^{(r)} E_{j}^{(s)}+E_{j}^{(s)} E_{i}^{(r)}\right), \\
{\left[F_{i}^{(r+1)}, F_{j}^{(s)}\right]-\left[F_{i}^{(r)}, F_{j}^{(s+1)}\right] } & =-\frac{1}{2} h\left(\alpha_{i}, \alpha_{j}\right)\left(F_{i}^{(r)} F_{j}^{(s)}+F_{j}^{(s)} F_{i}^{(r)}\right),
\end{aligned}
$$




$$
\begin{aligned}
\operatorname{sym}\left[E_{i}^{\left(r_{1}\right)},\left[E_{i}^{\left(r_{2}\right)}, \ldots\left[E_{i}^{\left(r_{N}\right)}, E_{j}^{(s)}\right] \ldots\right]\right] & =0 \quad \text { if } i \neq j \text { and } N=1-a_{i j} \\
\operatorname{sym}\left[F_{i}^{\left(r_{1}\right)},\left[F_{i}^{\left(r_{2}\right)}, \ldots\left[F_{i}^{\left(r_{N}\right)}, F_{j}^{(s)}\right] \ldots\right]\right] & =0 \quad \text { if } i \neq j \text { and } N=1-a_{i j}, \\
E_{\alpha_{i}} & =E_{i} \\
{\left[E_{\hat{\alpha}}^{(r)}, E_{\grave{\alpha}}^{(1)}\right] } & =h E_{\alpha}^{(r)} \\
F_{\alpha_{i}} & =F_{i}, \\
{\left[F_{\hat{\alpha}}^{(r)}, F_{\grave{\alpha}}^{(1)}\right] } & =h F_{\alpha}^{(r)} .
\end{aligned}
$$

We can repackage these generators and relations using generating series. Let

$$
E_{i}(u)=\sum_{s=1}^{\infty} E_{i}^{(s)} u^{-s}, \quad H_{i}(u)=1+\sum_{s=1}^{\infty} H_{i}^{(s)} u^{-s}, \quad F_{i}(u)=\sum_{s=1}^{\infty} F_{i}^{(s)} u^{-s} .
$$

Then the above relations can be written in series form. For example, the series version of the commutator relation between $E_{i}$ and $F_{i}$ is

$$
\left[E_{i}(u), F_{j}(v)\right]=-\delta_{i j} \frac{h}{u-v}\left(H_{i}(u)-H_{i}(v)\right) .
$$

Remark 3.6. Note that the Drinfeld Yangian $U_{h} \mathfrak{g}[t]$ and our Yangian $Y$ have natural $\mathbb{C}[h]$-forms; moreover, their $h=1$ specializations $U_{1} \mathfrak{g}[t]$ and $Y_{1}$ coincide as Hopf algebras. The gradings on $U_{h} \mathfrak{g}[t]$ and on $Y$ give rise to two different filtrations on $Y_{1}$. In the work of Brundan and Kleshchev [2006], these filtrations appear as the "loop filtration" and the "Kazhdan filtration", respectively.

3E. Identification of Yangian with functions of $G_{1} \llbracket t^{-1} \rrbracket$. From the results above, we can deduce that there is a perfect Hopf pairing between $U\left(t^{-1} \mathfrak{g} \llbracket t^{-1} \rrbracket\right)$ and $Y / h Y$, as per Corollary 3.4. Let us denote by $Q$ the root lattice for $\mathfrak{g}$, let $Q_{+}$denote the positive root cone, and let $Q_{>}=Q_{+} \backslash\{0\}$ and $Q_{<}=-Q_{>}$.

Lemma 3.7. The Drinfeld Yangian $U_{h} \mathfrak{g}[t], Y$, and $Y / h Y$ are all $Q$-graded Hopf algebras (all tensor products being graded by total degree). The pairing between $U\left(t^{-1} \mathfrak{g} \llbracket t^{-1} \rrbracket\right)$ and $Y / h Y$ respects this grading.

Proof. The Hopf grading on these spaces is induced by the action of the elements $h_{i}^{(0)}\left(\right.$ resp. $\left.H_{i}^{(1)}\right)$. In each case, coproducts preserve total degree since the coproduct is a homomorphism and the above elements are Lie algebra-like.

It is clear from Corollary 3.4 that the pairing between $U\left(t^{-1} \mathfrak{g} \llbracket t^{-1} \rrbracket\right)$ and $Y / h Y$ respects the grading for pairings $\langle y, x\rangle$ when $y \in t^{-1} \mathfrak{g} \llbracket\left[t^{-1} \rrbracket\right.$ and $x \in Y_{0}$. The result follows for monomials $y_{1} \ldots y_{k} \in U\left(t^{-1} \mathfrak{g} \llbracket t^{-1} \rrbracket\right)$ by induction on $k$.

For $\alpha \in Q$, let $Y(\alpha)$ be the corresponding component of $Y / h Y$ as per Lemma 3.7. 
Proposition 3.8. In $Y / h Y$ we have:

$$
\begin{aligned}
& \Delta\left(H_{i}^{(r)}\right)=H_{i}^{(r)} \otimes 1+1 \otimes H_{i}^{(r)}+\sum_{s=1}^{r-1} H_{i}^{(s)} \otimes H_{i}^{(r-s)}+\bigoplus_{\substack{\alpha+\beta=0 \\
\alpha \in Q_{<}, \beta \in Q_{>}}} Y(\alpha) \otimes Y(\beta), \\
& \Delta\left(E_{i}^{(r)}\right)=E_{i}^{(r)} \otimes 1+1 \otimes E_{i}^{(r)}+\sum_{s=1}^{r-1} H_{i}^{(s)} \otimes E_{i}^{(r-s)}+\bigoplus_{\substack{\alpha+\beta=\alpha_{i} \\
\alpha \in Q_{<}, \beta \in Q_{>}}} Y(\alpha) \otimes Y(\beta), \\
& \Delta\left(F_{i}^{(r)}\right)=F_{i}^{(r)} \otimes 1+1 \otimes F_{i}^{(r)}+\sum_{s=1}^{r-1} F_{i}^{(s)} \otimes H_{i}^{(r-s)}+\bigoplus_{\substack{\alpha+\beta=-\alpha_{i} \\
\alpha \in Q_{<}, \beta \in Q_{>}}} Y(\alpha) \otimes Y(\beta) .
\end{aligned}
$$

Proof. To begin, we recall that $\Delta\left(X^{(1)}\right)=X^{(1)} \otimes 1+1 \otimes X^{(1)}$ for all $x \in \mathfrak{g}$. Also, using the presentation of $U_{h} \mathfrak{g}[t]$ with generators $x, J(x)$ for $x \in \mathfrak{g}$ (for which the coproduct is known), a direct calculation yields

$$
\Delta\left(H_{i}^{(2)}\right)=H_{i}^{(2)} \otimes 1+1 \otimes H_{i}^{(2)}+H_{i}^{(1)} \otimes H_{i}^{(1)}-\sum_{\beta \in \Phi_{+}} C_{\beta}\left(\beta, \alpha_{i}\right) F_{\beta}^{(1)} \otimes E_{\beta}^{(1)},
$$

where $\left(e_{\beta}, f_{\beta}\right)=C_{\beta}^{-1}$. We prove the coproduct for $E_{i}^{(r)}$ by induction on $r$, using the identity

$$
E_{i}^{(r+1)}=\frac{1}{\left(\alpha_{i}, \alpha_{i}\right)}\left\{H_{i}^{(2)}, E_{i}^{(r)}\right\}-H_{i}^{(1)} E_{i}^{(r)} .
$$

The coproduct of the right side is expanded using the Poisson-Hopf algebra relations, the formula for $\Delta\left(H_{i}^{(2)}\right)$, and the inductive hypothesis. The above identity is then applied again to reduce the terms in the result, and yields the form as claimed.

An analogous induction proves the case of $\Delta\left(F_{i}^{(r)}\right)$. Finally, we take the coproduct of the identity

$$
H_{i}^{(r)}=\left\{E_{i}^{(r)}, F_{i}^{(1)}\right\}
$$

to finish the proof.

Recall that the pairing between $U\left(t^{-1} \mathfrak{g} \llbracket t^{-1} \rrbracket\right)$ and $Y / h Y$ is determined, as per Corollary 3.4, by the pairing between $t^{-1} \mathfrak{g} \llbracket t^{-1} \rrbracket$ and $\mathfrak{g}[t]$ given in Section 2F. Take an "FHE" total ordering on the generators $f_{\alpha} t^{r}, h_{i} t^{r}, e_{\alpha} t^{r}$ for $U\left(t^{-1} \mathfrak{g} \llbracket t^{-1} \rrbracket\right)$. Then it is easy to see that the previous lemma and proposition completely control the pairing between $U\left(t^{-1} \mathfrak{g} \llbracket t^{-1} \rrbracket\right)$ and $Y / h Y$ for the corresponding PBW basis. For example, $-F_{i}^{(r)}$ acts as the dual of the basis element $e_{i} t^{-r}$, etc.

Theorem 3.9. There is an isomorphism $\phi: Y / h Y \cong \mathcal{O}\left(G_{1} \llbracket t^{-1} \rrbracket\right)$ of $\mathbb{N}$-graded 
Poisson-Hopf algebras such that

$$
\begin{aligned}
\phi\left(H_{i}(u)\right) & =\prod_{j} \Delta_{\omega_{j}, \omega_{j}}(u)^{-a_{j i}}, \\
\phi\left(F_{i}(u)\right) & =d_{i}^{-1 / 2} \frac{\Delta_{\omega_{i}, s_{i} \omega_{i}}(u)}{\Delta_{\omega_{i}, \omega_{i}}(u)}, \\
\phi\left(E_{i}(u)\right) & =d_{i}^{-1 / 2} \frac{\Delta_{S_{i} \omega_{i}, \omega_{i}}(u)}{\Delta_{\omega_{i}, \omega_{i}}(u)},
\end{aligned}
$$

where $\mathcal{O}\left(G_{1} \llbracket t^{-1} \rrbracket\right)$ is graded using the loop rotation $\mathbb{C}^{\times}$action.

Proof. We check explicitly that the right-hand sides act as described by the previous proposition. Let $X=\left(x_{1} t^{r_{1}}\right) \ldots\left(x_{k} t^{r_{k}}\right) \in U\left(t^{-1} \mathfrak{g} \llbracket\left[t^{-1} \rrbracket\right)\right.$ be a basis monomial with the FHE order as chosen above. Then we have

$$
\begin{aligned}
& \frac{\Delta_{\omega_{i}, s_{i} \omega_{i}}(u)}{\Delta_{\omega_{i}, \omega_{i}}(u)}(X) \\
& \quad=-\left.d_{i}^{-1 / 2} \frac{\partial^{k}}{\partial z_{1} \ldots \partial z_{k}} \frac{\left\langle v_{-\omega_{i}},\left(1+z_{1} u^{r_{1}} x_{1}\right) \ldots\left(1+z_{k} u^{r_{k}} x_{k}\right) f_{i} v_{\omega_{i}}\right\rangle}{\left\langle v_{-\omega_{i}},\left(1+z_{1} u^{r_{1}} x_{1}\right) \ldots\left(1+z_{k} u^{r_{k}} x_{k}\right) v_{\omega_{i}}\right\rangle}\right|_{z_{1}=\ldots=z_{k}=0},
\end{aligned}
$$

noting that $\overline{s_{i}} v_{\omega_{i}}=f_{i}^{\prime} v_{\omega_{i}}=-d_{i}^{-1 / 2} f_{i} v_{\omega_{i}}$ in the generalized minor (see Section 2A). Since we have an FHE order, to get something nonzero in the right-hand numerator, $x_{k}$ must be a multiple of $e_{i}$, since $e_{i} f_{i} v_{\omega_{i}}=h_{i} v_{\omega_{i}}=d_{i} v_{\omega_{i}}$. In this case, $z_{k} u^{r_{k}} e_{i}$ does not contribute to the denominator, and the remaining factors cancel, leaving

$$
\frac{\Delta_{\omega_{i}, s_{i} \omega_{i}}(u)}{\Delta_{\omega_{i}, \omega_{i}}(u)}(X)=-\left.d_{i}^{1 / 2} \frac{\partial^{k}}{\partial z_{1} \ldots \partial z_{k}} z_{k} u^{r_{k}}\right|_{z_{1}=\cdots=z_{k}=0},
$$

so $X$ must have been $e_{i} t^{r}$ to start with. But this is precisely how $d_{i}^{1 / 2} F_{i}(u)$ acts on $X$. Similar computations hold in the two remaining cases.

To prove the equality for $H_{i}(u)$ one can also work in $\mathcal{O}\left(G_{1} \llbracket t^{-1} \rrbracket\right)$, and build off the known results $E_{i}(u)$ and $F_{i}(u)$, since we must have

$$
\frac{\phi\left(H_{i}(u)\right)-\phi\left(H_{i}(v)\right)}{u-v}=-\left\{\phi\left(E_{i}(u)\right), \phi\left(F_{i}(v)\right)\right\} .
$$

We can then use formula (1) and identities for generalized minors.

The nondegeneracy of both Hopf pairings implies that $\phi$ is an injection. It follows that $\phi$ is an isomorphism from a dimension count; both $Y / h Y$ and $\mathcal{O}\left(G_{1} \llbracket t^{-1} \rrbracket\right)$ have Hilbert series for the loop grading given by

$$
\prod_{i=1}^{\infty} \frac{1}{\left(1-q^{i}\right)^{\operatorname{dim} \mathfrak{g}}} .
$$


Indeed, for $Y / h Y$ this follows from the PBW theorem coming from $Y$, since $Y$ is a free $\mathbb{C} \llbracket h \rrbracket$-algebra. On the other hand, the Hilbert series on $\mathcal{O}\left(G_{1} \llbracket t^{-1} \rrbracket\right)$ is the same as the Hilbert series for $\operatorname{Sym}\left(t^{-1} \mathfrak{g} \llbracket t^{-1} \rrbracket\right)$, since as $G_{1} \llbracket t^{-1} \rrbracket$ is pro-unipotent, we have an isomorphism of vector spaces.

3F. Shifted Yangians. The Yangian has a very interesting class of subalgebras: the shifted Yangians. Let $\mu$ be a dominant coweight.

We will now redefine elements

$$
F_{\alpha}^{(s)}=\frac{1}{h}\left[F_{\hat{\alpha}}^{\left(s-\left\langle\mu^{*}, \check{\alpha}\right\rangle\right)}, F_{\check{\alpha}}^{\left(\left\langle\mu^{*}, \check{\alpha}\right\rangle+1\right)}\right]
$$

for $\alpha$ a positive nonsimple root and for $s>\left\langle\mu^{*}, \alpha\right\rangle$. Note that these $F_{\alpha}^{(s)}$ depend on $\mu$.

Definition 3.10. The shifted Yangian $Y_{\mu}$ is the subalgebra of $Y$ generated by $E_{\alpha}^{(s)}$ for all $\alpha, s, H_{i}^{(s)}$ for all $i, s$, and $F_{\alpha}^{(s)}$ for $s>\left\langle\mu^{*}, \alpha\right\rangle$.

Proposition 3.11. (1) Monomials in the $E_{\alpha}^{(s)}, H_{i}^{(s)}, F_{\alpha}^{(s)}$ give a basis for $Y_{\mu}$.

(2) The natural map $Y_{\mu} / h Y_{\mu} \rightarrow Y / h Y$ is injective.

Proof. We first construct a PBW basis for $Y$ slightly different from the one described in Section 3D. The generators $E_{\alpha}^{(s)}$ are defined as usual (see Section 3D). The generators $F_{\alpha}^{(s)}$ are given the usual definition when $s \leq\left\langle\mu^{*}, \alpha\right\rangle$, but for $s>\left\langle\mu^{*}, \alpha\right\rangle$ we take definition (4). By the general remarks following Theorem 3.3, ordered monomials in generators $F_{\alpha}^{(s)}, H_{i}^{(s)}, E_{\alpha}^{(s)}$ are a PBW basis of $Y$.

Any element $x \in Y_{\mu}$ can be expressed as a linear combination of these PBW monomials. We now show that any monomials appearing in such an expression do not contain factors of the form $F_{\alpha}^{(s)}$ for $s \leq\left\langle\mu^{*}, \alpha\right\rangle$.

By definition, $x$ is a linear combination of (unordered) monomials in $F_{\alpha}^{(s)}, H_{i}^{(t)}$, $E_{\alpha}^{(u)}$, where $s>\left\langle\mu^{*}, \alpha\right\rangle$. To put $x$ in PBW form, one has to commute these generators past each other. By definition, when $s>\left\langle\mu^{*}, \alpha\right\rangle, F_{\alpha}^{(s)}$ is a linear combination of monomials built from $F_{i}^{(t)}$, where $t>\left\langle\mu^{*}, \alpha_{i}\right\rangle$. Therefore it suffices to show that when commuting such $F_{i}^{(t)}$ past the other generators of $Y_{\mu}$ one never obtains factors of the form $F_{j}^{(u)}$ for $u \leq\left\langle\mu^{*}, \alpha_{j}\right\rangle$. This is a direct consequence of the relations appearing in Theorem 3.5.

This proves the first statement of the theorem. The second part is a direct consequence of the first.

In the limit as $\mu \rightarrow \infty$, we then obtain $Y_{\infty}$, which is the subalgebra generated by all $E_{\alpha}^{(s)}, A_{i}^{(s)}$. This is called the Borel Yangian in [Finkelberg and Rybnikov 2010].

We will now show that this shifted Yangian is a quantization of $\mathrm{Gr}_{\mu}$. Recall that $\mathrm{O}\left(\mathrm{Gr}_{\mu}\right)$ is embedded as a Poisson subalgebra of $\mathcal{O}\left(G_{1} \llbracket t^{-1} \rrbracket\right)$. 
Theorem 3.12. The isomorphism $\phi$ restricts to an isomorphism of Poisson algebras from $Y_{\mu} / h Y_{\mu}$ to $\mathcal{O}\left(\mathrm{Gr}_{\mu}\right)$.

Proof. First note that $Y_{\mu} / h Y_{\mu}$ is generated as a Poisson algebra by all $E_{i}^{(s)}, A_{i}^{(s)}$, and those $F_{i}^{(s)}$ for $i>\left\langle\mu^{*}, \alpha_{i}\right\rangle$. We note that Lemma 2.19 shows that the image of these generators under $\phi$ land in the subalgebra $\mathcal{O}\left(\mathrm{Gr}_{\mu}\right)$.

Since $\mathcal{O}\left(\mathrm{Gr}_{\mu}\right)$ is a Poisson subalgebra of $\mathcal{O}\left(G_{1} \llbracket t^{-1} \rrbracket\right)$, we see that $\phi$ restricts to a map $Y_{\mu} / h Y_{\mu} \rightarrow \mathcal{O}\left(G_{1} \llbracket t^{-1} \rrbracket\right)$. This map is injective, since it is the restriction of an injective map. Thus, we only need to show that it is surjective, which we do by a dimension count.

Note that by Lemma 2.3, the isotropy Lie algebra of $t^{w_{0} \mu}$ in $G_{1} \llbracket t^{-1} \rrbracket$ is the finite-dimensional nilpotent Lie algebra

$$
\bigoplus \bigoplus_{\alpha \in \Delta_{+}} \bigoplus_{i=1}^{-\left\langle w_{0} \mu, \alpha\right\rangle} t^{-i} \mathfrak{g}_{\alpha}
$$

As a $\mathbb{C}^{*}$-module, the functions on the group are identical to those on the Lie algebra by the unipotence of the stabilizer. Thus, if we let $d(k)$ be the number of roots such that $\left\langle w_{0} \mu, \alpha\right\rangle<k$, the Hilbert series of the functions on the stabilizer is

$$
\prod_{i=1}^{\infty} \frac{1}{\left(1-q^{i}\right)^{d(i)}} .
$$

The Hilbert series of $\mathcal{O}\left(G_{1} \llbracket t^{-1} \rrbracket\right)^{G_{1} \llbracket t^{-1} \rrbracket_{\mu}}$ is the quotient of that of $\mathcal{O}\left(G_{1} \llbracket t^{-1} \rrbracket\right)$ by that of functions on the stabilizer. That is, it is

$$
\prod_{i=1}^{\infty} \frac{1}{\left(1-q^{i}\right)^{\operatorname{dim} \mathfrak{g}-d(i)}} .
$$

On the other hand, the PBW basis for the shifted Yangian gives us the same Hilbert series for $Y_{\mu}$.

Thus the shifted Yangian $Y_{\mu}$ gives a quantization of $\mathrm{Gr}_{\mu}$.

Remark 3.13. We should note that it is this theorem that forces us to use the thick Grassmannian; it will fail if we take the analogue of $\mathrm{Gr}_{\mu}$ in the thin affine Grassmannian, since this has "too many" functions, and will correspond to a completion of $Y_{\mu}$.

3G. Deformation of the Yangian. We consider a deformation of the Yangian, which we think of as related to the Beilinson-Drinfeld Grassmannian deforming the affine Grassmannian. We consider for each node $i$ in the Dynkin diagram an infinite sequence of parameters $r_{i}^{(1)}, r_{i}^{(2)}, \ldots \in \mathbb{C} \llbracket h \rrbracket$ and their generating series $r_{i}(u)=1+r_{i}^{(1)} u^{-1}+\cdots$. 
Consider the algebra $Y(\boldsymbol{r})$ generated by the coefficients of $E_{i}(u), F_{i}(u), A_{i}(u)$. The relations are as in the previous section, with the relation (3) replaced by

$$
(u-v)\left[E_{i}(u), F_{i}(v)\right]=-h\left(r_{i}(u) H_{i}(u)-r_{i}(v) H_{i}(v)\right),
$$

and let $Y_{\mu}(\boldsymbol{r})$ be the shifted analogue of this algebra. $Y(\boldsymbol{r})$ is actually isomorphic to the trivial deformation of the Yangian via the map $H_{i}(u) \mapsto H_{i}(u) / r_{i}(u)$.

\section{Quantization of slices}

In order to quantize the slices $\mathrm{Gr}_{\mu}^{\bar{\lambda}}$, we will need to define a quotient of $Y_{\mu}$ (and its deformations $\left.Y_{\mu}(\boldsymbol{r})\right)$.

4A. Change of Cartan generators. It will be convenient for us to change the Cartan generators of $Y$. Following [Gerasimov et al. 2004], we define $A_{i}^{(s)}$ by the equation

$$
H_{i}(u)=\frac{\prod_{j \neq i} \prod_{p=1}^{-a_{j i}} A_{j}\left(u-\frac{h}{2}\left(\alpha_{i}+p \alpha_{j}, \alpha_{j}\right)\right)}{A_{i}(u) A_{i}\left(u-\frac{h}{2}\left(\alpha_{i}, \alpha_{i}\right)\right)},
$$

where $A_{i}(u)=1+\sum_{s=1}^{\infty} A_{i}^{(s)} u^{-s}$.

Example 4.1. In the $G=\mathrm{SL}_{2}$ case, this gives $H(u)=\frac{1}{A(u) A(u-h)}$, and so for example we have

$$
H^{(1)}=-2 A^{(1)}, \quad H^{(2)}=3 A^{(1)^{2}}-h A^{(1)}-2 A^{(2)} .
$$

Proposition 4.2 [Gerasimov et al. 2004, Lemma 2.1]. Equation (6) uniquely determines all the $A_{i}^{(s)}$.

One can think of the new generators $A_{i}^{(s)}$ as being related to the fundamental coweights of $G$, whereas the $H_{i}^{(s)}$ match with the simple coroots. In particular, we have the following result which follows by setting $h=0$ in (6).

Proposition 4.3. Let

$$
\phi: Y / h Y \rightarrow \mathcal{O}\left(G_{1} \llbracket t^{-1} \rrbracket\right)
$$

be the isomorphism from Theorem 3.9. Then $\phi\left(A_{i}^{(s)}\right)=\Delta_{\omega_{i}, \omega_{i}}^{(s)}$.

4B. The GKLO representation. In this section, we describe certain representations via difference operators of shifted Yangians, based on [Gerasimov et al. 2004]. Fix an orientation of the Dynkin diagram; we will write $i \leftarrow j$ to denote arrows in this quiver. This will replace the ordering on the simple roots in [loc. cit.].

Fix a dominant coweight $\lambda$ such that $\mu \leq \lambda$ and let $m_{i}=\left\langle\lambda-\mu, \omega_{i^{*}}\right\rangle$ and let $\lambda_{i}=\left\langle\lambda, \alpha_{i *}\right\rangle$. 
Define a $\mathbb{C} \llbracket h \rrbracket$-algebra $D_{\mu}^{\lambda}$, with generators $z_{i, k}, \beta_{i, k}, \beta_{i, k}^{-1}$, for $i \in I$ and $1 \leq$ $k \leq m_{i}$, and $\left(z_{i, k}-z_{i, l}\right)^{-1}$, and relations that all generators commute except that $\beta_{i, k} z_{i, k}=\left(z_{i, k}+d_{i} h\right) \beta_{i, k}$.

This algebra $D_{\mu}^{\lambda}$ is an algebra of $h$-difference operators.

Proposition 4.4. The algebra $D_{\mu}^{\lambda}$ is a free $\mathbb{C} \llbracket h \rrbracket$-algebra and we have an isomorphism of Poisson algebras

$$
D_{\mu}^{\lambda} / h D_{\mu}^{\lambda} \cong \mathbb{C}\left[z_{i, k},\left(z_{i, k}-z_{i, l}\right)^{-1}, \beta_{i, k}, \beta_{i, k}^{-1}\right],
$$

where the right-hand side is given the Poisson structure defined by $\left\{\beta_{i, k}, z_{i, k}\right\}=$ $d_{i} \beta_{i, k}$ and all other generators Poisson commute.

Proof. Obviously, we have a map

$$
\mathbb{C}\left[z_{i, k},\left(z_{i, k}-z_{i, l}\right)^{-1}, \beta_{i, k}, \beta_{i, k}^{-1}\right] \rightarrow D_{\mu}^{\lambda} / h D_{\mu}^{\lambda}
$$

by observing that $D_{\mu}^{\lambda} / h D_{\mu}^{\lambda}$ is commutative. From the Bergman diamond lemma, we see that the algebra $D_{\mu}^{\lambda}$ has a PBW basis consisting of

$$
h^{p} \cdot \prod \beta_{i, k}^{ \pm a_{i, k}} \cdot \prod z_{j, k}^{b_{j, k}} \cdot \prod_{k<\ell}\left(z_{i, k}-z_{i, \ell}\right)^{e_{i, k, \ell}}
$$

subject to the restriction that if $b_{j, k} \neq 0$, then $k$ must be maximal in its equivalence class for the relation given by the transitive closure of the binary relation $k \sim \ell$ if $e_{j, k, \ell} \neq 0$. Freeness over $\mathbb{C} \llbracket h \rrbracket$ follows immediately and since the same monomials give a basis of $\mathbb{C} \llbracket h \rrbracket\left[z_{i, k},\left(z_{i, k}-z_{i, l}\right)^{-1}, \beta_{i, k}, \beta_{i, k}^{-1}\right]$, this confirms that we have the desired isomorphism. The Poisson bracket calculation follows immediately from the relations.

Fix some complex numbers $c_{i}^{(r)}$ for $i \in I, 1 \leq r \leq \lambda_{i}$. For any variable $x$, consider the monic degree- $\lambda_{i}$ polynomial whose coefficients are the numbers $c_{i}^{(r)}$,

$$
C_{i}(x)=x^{\lambda_{i}}+c_{i}^{(1)} x^{\lambda_{i}-1}+\cdots+c_{i}^{\left(\lambda_{i}\right)} .
$$

Note that $x^{-\lambda_{i}} C_{i}(x)=1+c_{i}^{(1)} x^{-1}+\cdots+c_{i}^{\left(\lambda_{i}\right)} x^{-\lambda_{i}}$. We also introduce polynomials $Z_{i}(x)=\prod_{k=1}^{m_{i}}\left(x-z_{i, k}\right)$ and $Z_{i, k}(x)=\prod_{\ell \neq k}\left(x-z_{i, \ell}\right)$. Let $\mu_{i}=\left\langle\mu, \alpha_{i^{*}}\right\rangle$ and set $F_{\mu, i}(u)=\sum_{s=1}^{\infty} F_{i}^{\left(s+\mu_{i}\right)} u^{-s}$. Finally, for any $\boldsymbol{c}$ as above, define $\boldsymbol{r}$ by

$$
r_{i}(u)=u^{-\lambda_{i}} C_{i}(u) \frac{\prod_{j \neq i} \prod_{p=1}^{-a_{j i}}\left(1-u^{-1}\left(h d_{i} \frac{a_{i j}}{2}+h d_{j} p\right)\right)^{m_{j}}}{\left(1-h d_{i} u^{-1}\right)^{m_{i}}} .
$$

We are now ready to define the $G K L O$ representation: 
Theorem 4.5. There is a map of $\mathbb{C} \llbracket h \rrbracket-$ algebras, $\Psi_{\mu}^{\lambda}: Y_{\mu}(\boldsymbol{r}) \rightarrow D_{\mu}^{\lambda}$, defined by:

$$
\begin{aligned}
& A_{i}(u) \mapsto u^{-m_{i}} Z_{i}(u), \\
& E_{i}(u) \mapsto d_{i}^{-1 / 2} \sum_{k=1}^{m_{i}} \frac{\prod_{j \rightarrow i} \prod_{p=1}^{-a_{j i}} Z_{j}\left(z_{i, k}-h d_{i} \frac{a_{i j}}{2}-h d_{j} p\right)}{\left(u-z_{i, k}\right) Z_{i, k}\left(z_{i, k}\right)} \beta_{i, k}^{-1} .
\end{aligned}
$$

And $F_{\mu, i}(u)$ maps to

$$
-d_{i}^{-1 / 2} \sum_{k=1}^{m_{i}} C_{i}\left(z_{i, k}+h d_{i}\right) \frac{\prod_{j \leftarrow i} \prod_{p=1}^{-a_{j i}} Z_{j}\left(z_{i, k}-h d_{i}\left(\frac{a_{i j}}{2}-1\right)-h d_{j} p\right)}{\left(u-z_{i, k}-h d_{i}\right) Z_{i, k}\left(z_{i, k}\right)} \beta_{i, k} .
$$

Proof. When $\mu=0$, this is a reformulation of Theorem 3.1(i) of [Gerasimov et al. 2004]. Suppose then that $\mu \neq 0$. Then the proof of the theorem cited applies to all the relations in $Y_{\mu}$ except for the commutator relation between $E_{i}(u)$ and $F_{\mu, i}(v)$.

In the shifted Yangian this relation takes the form

$$
(u-v)\left[E_{i}(u), F_{\mu, i}(v)\right]=h\left(J_{\mu, i}(v)-J_{\mu, i}(u)\right),
$$

where $J_{i}(v)=r_{i}(v) H_{i}(v)=\sum_{p=0}^{\infty} J_{i}^{(p)} v^{-p}$ and

$$
J_{\mu, i}(v)=\sum_{p=1}^{\infty} J_{i}^{\left(p+\mu_{i}\right)} v^{-p} .
$$

To express the left-hand side of (8), we set

$$
\begin{aligned}
& L_{i}(v)=\frac{C_{i}\left(z_{i, k}+h d_{i}\right) \prod_{j \neq i} \prod_{p=1}^{-a_{j i}} Z_{j}\left(z_{i, k}-h d_{i}\left(\frac{a_{i j}}{2}-1\right)-h d_{j} p\right)}{Z_{i, k}\left(z_{i, k}+h d_{i}\right) Z_{i, k}\left(z_{i, k}\right)\left(v-z_{i, k}-h d_{i}\right)}, \\
& R_{i}(v)=\frac{C_{i}\left(z_{i, k}\right) \prod_{j \neq i} \prod_{p=1}^{-a_{j i}} Z_{j}\left(z_{i, k}-h d_{i} \frac{a_{i j}}{2}-h d_{j} p\right)}{Z_{i, k}\left(z_{i, k}-h d_{i}\right) Z_{i, k}\left(z_{i, k}\right)\left(v-z_{i, k}\right)} .
\end{aligned}
$$

Then the left-hand side of (8) is equal to

$$
d_{i}^{-1} \sum_{k=1}^{m_{i}}\left(L_{i}(v)-R_{i}(v)\right)-\left(L_{i}(u)-R_{i}(u)\right) .
$$

Note that we expressed this sum as a " $v$-part" minus a " $u$-part". 
Now we consider the right-hand side of (8). Note that

$$
\lambda_{i}=\mu_{i}+2 m_{i}+\sum_{j \leftrightarrow i} a_{j i} m_{j} .
$$

Therefore,

$$
r_{i}(u)=u^{-\mu_{i}} \frac{C_{i}(u) \prod_{j \neq i} \prod_{p=1}^{-a_{j i}}\left(u-h d_{i} \frac{a_{i j}}{2}-h d_{j} p\right)^{m_{j}}}{u^{m_{i}}\left(u-h d_{i}\right)^{m_{i}}} .
$$

Now

$$
H_{i}(u) \mapsto \frac{u^{m_{i}}\left(u-h d_{i}\right)^{m_{i}}}{\prod_{j \neq i} \prod_{p=1}^{-a_{j i}}\left(u-h d_{i} \frac{a_{i j}}{2}-h d_{j} p\right)^{m_{j}}} \frac{\prod_{j \neq i} \prod_{p=1}^{-a_{j i}} Z_{j}\left(u-h d_{i} \frac{a_{i j}}{2}-h d_{j} p\right)}{Z_{i}(u) Z_{i}\left(u-h d_{i}\right)},
$$

and hence

$$
r_{i}(u) H_{i}(u) \mapsto u^{-\mu_{i}} C_{i}(u) \frac{\prod_{j \neq i} \prod_{p=1}^{-a_{j i}} Z_{j}\left(u-h d_{i} \frac{a_{i j}}{2}-h d_{j} p\right)}{Z_{i}(u) Z_{i}\left(u-h d_{i}\right)} .
$$

Therefore

$$
C_{i}(u) \frac{\prod_{j \neq i} \prod_{p=1}^{-a_{j i}} Z_{j}\left(u-h d_{i} \frac{a_{i j}}{2}-h d_{j} p\right)}{Z_{i}(u) Z_{i}\left(u-h d_{i}\right)}=\sum_{p=0}^{\infty} J_{i}^{(p)} u^{\mu_{i}-p} .
$$

On the other hand,

$$
J_{\mu, i}(u)=\sum_{p=\mu_{i}+1}^{\infty} J_{i}^{(p)} u^{\mu_{i}-p}
$$

showing that $J_{\mu, i}(u)$ is a truncation of

$$
C_{i}(u) \frac{\prod_{j \neq i} \prod_{p=1}^{-a_{j i}} Z_{j}\left(u-h d_{i} \frac{a_{i j}}{2}-h d_{j} p\right)}{Z_{i}(u) Z_{i}\left(u-h d_{i}\right)} .
$$

More precisely, for $r=1,2, \ldots$,

$$
\left.h J_{\mu, i}(u)\right|_{u^{-r}}=\left.h C_{i}(u) \frac{\prod_{j \neq i} \prod_{p=1}^{-a_{j i}} Z_{j}\left(u-h d_{i} \frac{a_{i j}}{2}-h d_{j} p\right)}{Z_{i}(u) Z_{i}\left(u-h d_{i}\right)}\right|_{u^{-r}} .
$$


Using partial fractions, we have that $\frac{h}{Z_{i}(u) Z_{i}\left(u-h d_{i}\right)}$ equals

$$
\sum_{k=1}^{m_{i}} \frac{1}{Z_{i k}\left(z_{i k}\right) Z_{i k}\left(z_{i k}+h d_{i}\right)\left(u-z_{i k}-h d_{i}\right)}-\frac{1}{Z_{i k}\left(z_{i k}\right) Z_{i k}\left(z_{i k}-h d_{i}\right)\left(u-z_{i k}\right)} .
$$

Therefore for $r=1,2, \ldots$, the $u^{-r}$-coefficient of $h J_{\mu, i}(u)$ is equal to the $u^{-r}$ coefficient of

$$
\begin{aligned}
\sum_{k=1}^{m_{i}} \frac{C_{i}(u) \prod_{j \neq i} \prod_{p=1}^{-a_{j i}} Z_{j}\left(u-h d_{i} \frac{a_{i j}}{2}-h d_{j} p\right)}{Z_{i k}\left(z_{i k}\right) Z_{i k}\left(z_{i k}+h d_{i}\right)\left(u-z_{i k}-h d_{i}\right)} & \\
- & \frac{C_{i}(u) \prod_{j \neq i} \prod_{p=1}^{-a_{j i}} Z_{j}\left(u-h d_{i} \frac{a_{i j}}{2}-h d_{j} p\right)}{Z_{i k}\left(z_{i k}\right) Z_{i k}\left(z_{i k}-h d_{i}\right)\left(u-z_{i k}\right)} .
\end{aligned}
$$

Now observe that for any polynomial $p(u)$ and for $r=1,2, \ldots$,

$$
\left.\frac{p(u)}{u-z}\right|_{u^{-r}}=\left.\frac{p(z)}{u-z}\right|_{u^{-r}} .
$$

Therefore for $r=1,2, \ldots$, the $u^{-r}$-coefficient of $h u^{\mu_{i}} J_{\mu, i}(u)$ is equal to the $u^{-r}$ coefficient of $\sum_{k=1}^{m_{i}} L_{i}(u)-R_{i}(u)$, proving (8).

Example 4.6. If $\mathfrak{g}=\mathfrak{s l}_{2}$ and $\lambda=\alpha^{\vee}, \mu=0$, then the formulas above simplify considerably. In this case,

$$
A(u) \mapsto 1-z u^{-1}, \quad E(u) \mapsto \frac{1}{u-z} \beta^{-1},
$$

and $F(u) \mapsto-\left((z+h)^{2}+c^{(1)}(z+h)+c^{(2)}\right) \frac{1}{u-z-h} \beta$. In particular,

$$
H^{(1)} \mapsto 2 z, \quad E^{(1)} \mapsto \beta^{-1}, \quad F^{(1)} \mapsto-\left((z+h)^{2}+c^{(1)}(z+h)+c^{(2)}\right) \beta .
$$

Restrict this representation to the copy of $\mathfrak{s l}_{2}$ generated by $E^{(1)}, H^{(1)}+c^{(1)}+h, F^{(1)}$, and consider these as difference operators acting on the polynomial ring $\mathbb{C}[z]$. (More precisely, these act on $\mathbb{C}[h \rrbracket[z]$, but one can specialize $h$ to 1.) This is a standard Whittaker module for $\mathfrak{s l}_{2}$ with generic nilpotent character.

Remark 4.7. We can define a $\mathbb{Z}$-grading on $D_{\mu}^{\lambda}$ by setting

$$
\operatorname{deg} h=1, \quad \operatorname{deg} z_{i, k}=1, \quad \operatorname{deg} \beta_{i, k}=m_{i}+\sum_{i \rightarrow j} a_{i j} m_{j}+\lambda_{i}-\mu_{i} .
$$

With this definition, the GKLO representation preserves grading. 
4C. Quantization of the slices $\mathrm{Gr}_{\mu}^{\bar{\lambda}}$. For any $\boldsymbol{c}$ as above, let $Y_{\mu}^{\lambda}(\boldsymbol{c})$ be the image of $Y_{\mu}(\boldsymbol{r})$ in $D_{\mu}^{\lambda}$ under the GKLO representation $\Psi_{\mu}^{\lambda}$ and let $I_{\mu}^{\lambda}(\boldsymbol{c})$ denote the kernel of $\Psi_{\mu}^{\lambda}$ (here $\boldsymbol{r}$ is determined from $\boldsymbol{c}$ by (7)).

Note that $Y_{\mu}^{\lambda}(\boldsymbol{c})$ is free as a $\mathbb{C} \llbracket h \rrbracket$-algebra since it is a subalgebra of $D_{\mu}^{\lambda}$, a free $\mathbb{C} \llbracket h \rrbracket$-algebra.

We have the isomorphism $Y_{\mu}(\boldsymbol{c}) \rightarrow Y_{\mu}$ from Section $3 \mathrm{G}$ and thus we get an isomorphism of Poisson algebras $Y_{\mu}(\boldsymbol{c}) / h Y_{\mu}(\boldsymbol{c}) \rightarrow \mathcal{O}\left(\mathrm{Gr}_{\mu}\right)$ from Theorem 3.12. On the other hand, because $Y_{\mu}^{\lambda}(\boldsymbol{c})$ is free as a $\mathbb{C} \llbracket h \rrbracket$-algebra, we get a surjection of Poisson algebras $Y_{\mu}(\boldsymbol{c}) / h Y_{\mu}(\boldsymbol{c}) \rightarrow Y_{\mu}^{\lambda}(\boldsymbol{c}) / h Y_{\mu}^{\lambda}(\boldsymbol{c})$.

We will now establish the following theorem, which shows that $Y_{\mu}^{\lambda}$ is a quantization of scheme supported on $\mathrm{Gr}_{\mu}^{\bar{\lambda}}$.

Theorem 4.8. There is a surjective map of Poisson algebras $Y_{\mu}^{\lambda}(\boldsymbol{c}) / h Y_{\mu}^{\lambda}(\boldsymbol{c}) \rightarrow$ $\mathcal{O}\left(\mathrm{Gr}_{\mu}^{\bar{\lambda}}\right)$ which is an isomorphism modulo the nilradical of the left-hand side.

Remark 4.9. Consider the map

$$
Y_{\mu}^{\lambda}(\boldsymbol{c}) / h Y_{\mu}^{\lambda}(\boldsymbol{c}) \rightarrow \mathbb{C}\left[z_{i, k},\left(z_{i, k}-z_{i, l}\right)^{-1}, \beta_{i, k}, \beta_{i, k}^{-1}\right]
$$

obtained by reducing the GKLO representation $\bmod h$. If we knew that this map was injective, then we would know that $Y_{\mu}^{\lambda}(\boldsymbol{c}) / h Y_{\mu}^{\lambda}(\boldsymbol{c})$ was reduced and that the map from Theorem 4.8 was an isomorphism. We will in fact make a stronger conjecture.

If Conjecture 2.20 holds, then we can strengthen Theorem 4.8 as follows.

Theorem 4.10. If Conjecture 2.20 holds, then:

(1) There is an isomorphism of Poisson algebras $Y_{\mu}^{\lambda}(\boldsymbol{c}) / h Y_{\mu}^{\lambda}(\boldsymbol{c}) \rightarrow \mathrm{O}\left(\mathrm{Gr}_{\mu}^{\bar{\lambda}}\right)$.

(2) $Y_{\mu}^{\lambda}(\boldsymbol{c})$ is the quotient of $Y_{\mu}(\boldsymbol{c})$ by the 2-sided ideal generated by $A_{i}^{(s)}$ for $s>m_{i}, i \in I$.

Proof of Theorem 4.8. Via the isomorphism $Y_{\mu}(\boldsymbol{c}) / h Y_{\mu}(\boldsymbol{c}) \rightarrow \mathrm{O}\left(\mathrm{Gr}_{\mu}\right)$, we can regard $Y_{\mu}^{\lambda}(\boldsymbol{c}) / h Y_{\mu}^{\lambda}(\boldsymbol{c})$ as a quotient of $\mathcal{O}\left(\mathrm{Gr}_{\mu}\right)$ by an ideal, which we denote by $I_{2}$.

First, note that $\Psi_{\mu}^{\lambda}\left(A_{i}^{(s)}\right)=0$ for $i \in I, s>m_{i}$, and thus $\Delta_{\omega_{i}, \omega_{i}}^{(s)} \in I_{2}$ for $i \in I$ and $s>m_{i}$. Since $I_{2}$ is a Poisson ideal, we see that $J_{\mu}^{\lambda} \subset I_{2}$.

By Proposition 2.21, we see that the vanishing locus of $J_{\mu}^{\lambda}$ is $\mathrm{Gr}_{\mu}^{\bar{\lambda}}$, and thus the vanishing locus of $I_{2}$ is contained in $\mathrm{Gr}_{\mu}^{\bar{\lambda}}$. Thus it suffices to show that the vanishing locus of $I_{2}$ is not strictly contained in $\mathrm{Gr}_{\mu}^{\bar{\lambda}}$.

Since $I_{2}$ is a Poisson ideal, we see that $V\left(I_{2}\right)$ is a Poisson subvariety of $\mathrm{Gr}_{\mu}^{\bar{\lambda}}$ and thus is the union of $\mathrm{Gr}_{\mu}^{\bar{\nu}}$, for $v \leq \lambda$. Suppose that we have

$$
V\left(I_{2}\right)=\bigcup_{j} \operatorname{Gr}_{\mu}^{\bar{j}_{j}}
$$


for $v_{j}<\lambda$. For each $j$, there exists $i$ such that $\left\langle v_{j}-\mu, \omega_{i^{*}}\right\rangle<\left\langle\lambda-\mu, \omega_{i^{*}}\right\rangle=m_{i}$. Thus applying Proposition 2.4, $\prod_{i} \Delta_{\omega_{i}, \omega_{i}}^{\left(m_{i}\right)}$ vanishes on $\bigcup_{j} \operatorname{Gr}_{\mu}^{\bar{v}_{j}}$. Hence for some $k$, we have $\left(\prod_{i} \Delta_{\omega_{i}, \omega_{i}}^{\left(m_{i}\right)}\right)^{k} \in I_{2}$.

On the other hand, we see that under the GKLO representation

$$
\Psi_{\mu}^{\lambda}\left(A_{i}^{\left(m_{i}\right)}\right)=(-1)^{m_{i}} z_{i, 1} \ldots z_{i, m_{i}},
$$

and thus under the map

$$
\mathcal{O}\left(\mathrm{Gr}_{\mu}\right) \cong Y_{\mu}(\boldsymbol{c}) / h Y_{\mu}(\boldsymbol{c}) \rightarrow D_{\mu}^{\lambda} / h D_{\mu}^{\lambda} \cong \mathbb{C}\left[z_{i, k},\left(z_{i, k}-z_{i, l}\right)^{-1}, \beta_{i, k}, \beta_{i, k}^{-1}\right],
$$

$\left(\prod_{i} \Delta_{\omega_{i}, \omega_{i}}^{\left(m_{i}\right)}\right)^{k}$ is mapped to a monomial in the $z_{i, k}$. In particular, this shows that $\left(\prod_{i} \Delta_{\omega_{i}, \omega_{i}}^{\left(m_{i}\right)}\right)^{k}$ does not lie in $I_{2}$, contradicting the previous paragraph.

Thus we conclude that $V\left(I_{2}\right)=\mathrm{Gr}_{\mu}^{\lambda}$ as desired.

Proof of Theorem 4.10. Suppose $I_{1}$ is the ideal of $\mathrm{Gr}_{\mu}^{\bar{\lambda}}$ in $\mathcal{O}\left(\mathrm{Gr}_{\mu}\right)$.

Let $K$ be the ideal in $Y_{\mu}(\boldsymbol{c})$ generated by $A_{i}^{(s)}$ for $s>m_{i}, i \in I$. Then we have an inclusion $K \subset I_{\mu}^{\lambda}(\boldsymbol{c})$ and a resulting map

$$
K / h K \rightarrow I_{\mu}^{\lambda}(\boldsymbol{c}) / h I_{\mu}^{\lambda}(\boldsymbol{c})=I_{2}
$$

which may not be injective. Let $I_{3}$ denote the image of this map. From the definitions, we see that $I_{3} \subset I_{2}$. Moreover, we have that $J_{\mu}^{\lambda} \subset I_{3}$, since $I_{3}$ is a Poisson ideal and it contains the generators of $I_{3}$.

In the previous proof we showed that $I_{2} \subset I_{1}$. Thus we have a chain of inclusions $J_{\mu}^{\lambda} \subset I_{3} \subset I_{2} \subset I_{1}$. On the other hand, Conjecture 2.20 shows us that $I_{1}=J_{\mu}^{\lambda}$.

Hence we conclude that $I_{1}=I_{2}=I_{3}=J_{\mu}^{\lambda}$. So the first assertion holds.

For the second assertion, note that $I_{3}=I_{2}$ implies that $K / h K \rightarrow I_{\mu}^{\lambda}(\boldsymbol{c}) / h I_{\mu}^{\lambda}(\boldsymbol{c})$ is surjective. Let $L=I_{\mu}^{\lambda}(\boldsymbol{c}) / K$. The long exact sequence for $\otimes_{\mathbb{C} \llbracket h \rrbracket} \mathbb{C}$ gives

$$
K / h K \rightarrow I_{\mu}^{\lambda}(\boldsymbol{c}) / h I_{\mu}^{\lambda}(\boldsymbol{c}) \rightarrow L / h L \rightarrow 0,
$$

and thus $L / h L=0$. By Nakayama's lemma, we conclude that $L=0$, and thus $K=I_{\mu}^{\lambda}(\boldsymbol{c})$ as desired.

4D. Universality of the quantization. There is already a rich literature on the theory of deformation quantizations of symplectic varieties. The most relevant work for us is [Bezrukavnikov and Kaledin 2004], showing the existence and uniqueness of deformation quantizations of symplectic resolutions. This theory can be applied directly to a smooth convolution variety $\operatorname{Gr}_{\mu}^{\vec{\lambda}}$. Moreover, as noted by Braden, Proudfoot and Webster [Braden et al. 2012, 3.4], it can be extended in a very straightforward way to the nonsmooth case $\operatorname{Gr}_{\mu}^{\vec{\lambda}}$, since we know that $\mathrm{Gr}_{\mu}^{\overrightarrow{\vec{\lambda}}}$ is a terminalization (Theorem 2.7). 
This shows that the variety $\mathrm{Gr}_{\mu}^{\bar{\lambda}}$ has a canonical family of quantizations which extend to a deformation quantization sheaf on $\operatorname{Gr}_{\mu}^{\vec{\lambda}}$. The base of this family is the same as the base for the universal deformation of $\mathrm{Gr}_{\mu}^{\bar{\lambda}}$ as a symplectic singularity (as constructed by Kaledin and Verbitsky [2002] or Namikawa [2011]). By [Namikawa $2010,1.1]$, this base $\mathbb{B}$ is an affine space modulo the action of a finite group. This group can be described by looking at the codimension-2 strata of the product of $\mathrm{Gr}_{\mu}^{\bar{\lambda}}$, which are $\mathrm{Gr}_{\mu}^{\lambda-\alpha_{i}}$, and taking the product of the Weyl groups attached to them by the McKay correspondence, which (using Example 2.2) in our case results in the symmetric groups $S_{\lambda, \mu}=\prod_{i: m_{i}>0} S_{\lambda_{i}}$. Here we use the fact that these strata are simply connected.

For the remainder of this section, let us regard the complex numbers $r_{i}^{(s)}$ and $c_{i}^{(s)}$ as variables and let $\tilde{Y}_{\mu}$ be the $\mathbb{C}\left[r_{i}^{(s)}\right]$-algebra which recovers the old $Y_{\mu}(\boldsymbol{r})$ upon specializing the variables. Let

$$
\tilde{Y}_{\mu}^{\lambda}=\tilde{Y}_{\mu} \otimes_{\mathbb{C}\left[r_{i}^{(s)}\right]} \mathbb{C}\left[c_{i}^{(s)}\right] /\left(\left\{A_{i}^{(s)} \mid s>m_{i}\right\}\right)
$$

(here we use a map $\mathbb{C}\left[r_{i}^{(s)}\right] \rightarrow \mathbb{C}\left[c_{i}^{(s)}\right]$ given by (7)). If Conjecture 2.20 (and hence Theorem 4.10) holds, then $\tilde{Y}_{\mu}^{\lambda}$ can be specialized (via a map $\mathbb{C}\left[c_{i}^{(s)}\right] \rightarrow \mathbb{C}$ ) to each of the $Y_{\mu}^{\lambda}(\boldsymbol{c})$. We conjecture that $\tilde{Y}_{\mu}^{\lambda}$ is related to the above universal quantization as follows.

First note that the $\mathrm{BD}$ analogue $\mathrm{Gr}_{\mu ; \mathrm{A}^{\rho(\lambda)}}^{\overline{\bar{\lambda}}}$ is a symplectic deformation of $\mathrm{Gr}_{\mu}^{\bar{\lambda}}$ over the base $A^{\rho(\lambda)}$, and thus is the pull-back of the universal deformation by a map $b: \mathbb{A}^{\rho(\lambda)} \rightarrow \mathbb{B}$.

Conjecture 4.11. (1) The map $b: \mathbb{A}^{\rho \lambda} \rightarrow \mathbb{B}$ descends to a surjective map $\tilde{b}:$ $\mathbb{A}^{\rho \lambda} / S_{\lambda, \mu} \rightarrow \mathbb{B}$.

(2) The algebra $\tilde{Y}_{\mu}^{\lambda}$ is the base change along $\tilde{b}$ of the universal, BezrukavnikovKaledin-type quantization.

Example 4.12. We continue Example 4.6, so $G=\mathrm{SL}_{2}$ and $\lambda=\alpha^{\vee}, \mu=0$. Note that in $Y_{\mu}^{\lambda}$, we have that $E^{(s)}=\left(-A^{(1)}\right)^{s-1} E^{(1)}$, and $F^{(s)}=F^{(1)}\left(-A^{(1)}\right)^{s-1}$, and so $Y_{\mu}^{\lambda}$ is generated by $E^{(1)}$ and $F^{(1)}$.

Let $U_{h} \mathfrak{s l}_{2}$ denote the $h$-version of the universal enveloping algebra of $\mathfrak{s l}_{2}$. Let $C=E F+F E+\frac{1}{2} H^{2}$ be its Casimir element. For any complex number $c$, let $Z_{c}$ denote the ideal in $U_{h} \mathfrak{s l}_{2}$ generated by the central element $C-c$. Standard results give that $U_{h} \mathfrak{s l}_{2} / Z_{c}$ is a quantization of the nilpotent cone of $\mathfrak{s l}_{2}$, which is isomorphic as a Poisson variety to $\mathrm{Gr}_{\mu}^{\bar{\lambda}}$.

The map

$$
E^{(1)} \mapsto E, \quad H^{(1)} \mapsto H+c^{(1)}+h, \quad F^{(1)} \mapsto F
$$


defines an isomorphism $Y_{\mu}^{\lambda} \cong U_{h} \mathfrak{s l}_{2} / Z_{c}$, where $c=2 c^{(2)}-\frac{1}{2}\left(c^{(1)}\right)^{2}+\frac{1}{2} h^{2}$. If we don't specialize, then the same formulas combined with the assignment

$$
c^{(2)} \mapsto-\frac{1}{2} C+\frac{1}{4}\left(c^{(1)}\right)^{2}-\frac{1}{4} h^{2}
$$

give an isomorphism

$$
\tilde{Y}_{0}^{2} \cong U_{h}\left(\mathfrak{s l}_{2}\right)\left[c^{(1)}\right] .
$$

In this example, $U_{h}\left(\mathfrak{s l}_{2}\right)$ is the universal quantization and $c^{(1)}$ a trivial deformation parameter. The universal family is

$$
\mathfrak{s l}_{2} \stackrel{\operatorname{tr}\left(a^{2}\right)}{\longrightarrow} \mathbb{C} .
$$

Since the fiber of the BD analogue over $(x, y) \in \mathbb{A}^{2}$ can be identified with matrices with eigenvalues $x$ and $y$, the map $b$ is just $b(x, y)=\frac{1}{4}(x-y)^{2}$. Thus, choosing $x+y$ and $(x-y)^{2}$ as generators of symmetric functions, $\tilde{b}$ is just the projection $\operatorname{map} \mathbb{A}^{2} \rightarrow \mathbb{A}^{1}$.

The sum of the $c_{i}^{(1)}$ is always a trivial deformation parameter; usually this is the only such parameter, but there are degenerate cases where other parameters can be trivialized as well (for example, if $\lambda=\mu$ ).

4E. Quantization of Zastava spaces. Here we assume that Conjecture 2.20 holds, and thus we will assume the conclusions of Theorem 4.10.

Let us fix $v$ in the positive coroot cone. Choose some $\mu_{0}$ such that $\mu_{0}+v$ is dominant. Let $\boldsymbol{c}$ be a collection of complex numbers as above and consider $Y_{\mu_{0}}^{\mu_{0}+v}(\boldsymbol{c})$.

Now for any dominant $\mu$ with $\mu \geq \mu_{0}$, we extend $c$ by 0 and (slightly abusing notation) consider $Y_{\mu}^{\mu+v}(\boldsymbol{c})$. Since the generators of $Y_{\mu}^{\mu+v}(\boldsymbol{c})$ are a subset of the generators of $Y_{\mu_{0}}^{\mu_{0}+v}(\boldsymbol{c})$ and the relations are the same, we obtain a map $Y_{\mu}^{\mu+v}(\boldsymbol{c}) \rightarrow$ $Y_{\mu_{0}}^{\mu_{0}+v}(\boldsymbol{c})$. It is easy to see that this map is an isomorphism on the $N$-th filtered piece if $\left\langle\mu, \alpha_{i}\right\rangle \geq N$ for all $i$.

Thus this system stabilizes to the algebra $Y_{\infty}^{\infty+v}$, which is the quotient of the Borel Yangian $Y_{\infty}$ by the 2-sided ideal generated by $A_{i}^{(s)}$ for $s>\left\langle v, \alpha_{i}\right\rangle$; perhaps surprisingly, this limit doesn't depend on $\boldsymbol{c}$ or our starting $\mu_{0}$.

Combining Theorem 4.10 with Theorem 2.10, we obtain the following (dependent on Conjecture 2.20), which was conjectured in [Finkelberg and Rybnikov 2010] for $G=\mathrm{SL}_{n}$ (and proven for $G=\mathrm{SL}_{2}$ ).

Theorem 4.13. $Y_{\infty}^{\infty+v} / h Y_{\infty}^{\infty+v}$ is isomorphic to the Poisson algebra $\mathrm{O}\left(Z_{v}\right)$.

Remark 4.14. As mentioned above, the GKLO representation gives rise to a map of graded Poisson algebras

$$
Y_{\mu}^{\lambda}(\boldsymbol{c}) / h Y_{\mu}^{\lambda}(\boldsymbol{c}) \rightarrow D_{\mu}^{\lambda}(\boldsymbol{c}) / h D_{\mu}^{\lambda}(\boldsymbol{c})
$$


(which we expect is an inclusion) and thus to a $\mathbb{C}^{\times}$-equivariant map of Poisson varieties

$$
\prod_{i}\left(\mathbb{C}^{m_{i}} \backslash \Delta\right) \times\left(\mathbb{C}^{\times}\right)^{m_{i}} \rightarrow \mathrm{Gr}_{\mu}^{\lambda}
$$

which we expect to be étale.

If we then compose with the map $G r_{\mu}^{\lambda} \rightarrow Z_{\lambda-\mu}$, we obtain $\prod_{i}\left(\mathbb{C}^{m_{i}} \backslash \Delta\right) \rightarrow Z_{\lambda-\mu}$, which was studied in [Gerasimov et al. 2004].

\section{Acknowledgements}

We would like to thank Alexander Braverman, Pavel Etingof, Mikhail Finkelberg, Ivan Mirković, Sergey Oblezin, Travis Schedler and Catharina Stroppel for extremely useful conversations. We also thank the referees for their careful reading of this paper.

\section{References}

[Beauville 2000] A. Beauville, "Symplectic singularities", Invent. Math. 139:3 (2000), 541-549. MR 2001f:14008 Zbl 0958.14001

[Beilinson et al. 1996] A. Beilinson, V. Ginzburg, and W. Soergel, "Koszul duality patterns in representation theory”, J. Amer. Math. Soc. 9:2 (1996), 473-527. MR 96k:17010 Zbl 0864.17006

[Bezrukavnikov and Kaledin 2004] R. Bezrukavnikov and D. Kaledin, "Fedosov quantization in algebraic context”, Mosc. Math. J. 4:3 (2004), 559-592, 782. MR 2006j:53130 Zbl 1074.14014

[Braden et al. 2012] T. Braden, N. Proudfoot, and B. Webster, "Quantizations of conical symplectic resolutions, I: Local and global structure”, preprint, 2012. arXiv 1208.3863

[Braden et al. $\geq 2014]$ T. Braden, A. Licata, N. Proudfoot, and B. Webster, "Quantizations of conical symplectic resolutions, II: Category O", in preparation.

[Braverman and Finkelberg 2011] A. Braverman and M. Finkelberg, "Semi-infinite Schubert varieties and quantum $K$-theory of flag manifolds", preprint, 2011. arXiv 1111.2266

[Brundan and Kleshchev 2006] J. Brundan and A. Kleshchev, "Shifted Yangians and finite Walgebras", Adv. Math. 200:1 (2006), 136-195. MR 2006m:17010 Zbl 1083.17006

[Brundan and Kleshchev 2008] J. Brundan and A. Kleshchev, "Representations of shifted Yangians and finite $W$-algebras”, Mem. Amer. Math. Soc. 196:918 (2008), viii+107. MR 2009i:17020 Zbl 1169.17009

[Drinfeld 1987] V. G. Drinfeld, "Quantum groups", pp. 798-820 in Proceedings of the International Congress of Mathematicians (Berkeley, CA), vol. 1-2, edited by A. M. Gleason, Amer. Math. Soc., Providence, RI, 1987. MR 89f:17017 Zbl 0667.16003

[Drinfeld 1993] V. G. Drinfeld, “On Poisson homogeneous spaces of Poisson-Lie groups", Teoret. Mat. Fiz. 95:2 (1993), 226-227. In Russian; translated in Theoret. and Math. Phys. 95:2 (1993), 524-535. MR 94k:58045 Zbl 0852.22018

[Etingof and Kazhdan 1998] P. Etingof and D. Kazhdan, "Quantization of Lie bialgebras, III", Selecta Math. (N.S.) 4:2 (1998), 233-269. MR 2000i:17033 Zbl 0915.17009

[Evens and Mirković 1999] S. Evens and I. Mirković, "Characteristic cycles for the loop Grassmannian and nilpotent orbits”, Duke Math. J. 97:1 (1999), 109-126. MR 2000h:22017 Zbl 1160.22306 
[Finkelberg and Mirković 1999] M. Finkelberg and I. Mirković, "Semi-infinite flags, I: Case of global curve $\boldsymbol{P}^{1}$ ", pp. 81-112 in Differential topology, infinite-dimensional Lie algebras, and applications, edited by A. Astashkevich and S. Tabachnikov, Amer. Math. Soc. Transl. Ser. 2 194, Amer. Math. Soc., Providence, RI, 1999. MR 2001j:14029 Zbl 1076.14512

[Finkelberg and Rybnikov 2010] M. Finkelberg and L. Rybnikov, "Quantization of Drinfeld Zastava", preprint, 2010. arXiv 1009.0676

[Finkelberg et al. 1999] M. Finkelberg, A. Kuznetsov, N. Markarian, and I. Mirković, "A note on a symplectic structure on the space of G-monopoles", Comm. Math. Phys. 201:2 (1999), 411-421. MR 2000g:53095 Zbl 0981.53083

[Gavarini 2002] F. Gavarini, “The quantum duality principle”, Ann. Inst. Fourier (Grenoble) 52:3 (2002), 809-834. MR 2003d:17016 Zbl 1054.17011

[Gavarini 2007] F. Gavarini, “The global quantum duality principle”, J. Reine Angew. Math. 612 (2007), 17-33. MR 2008k:17015 Zbl 1204.17010

[Gerasimov et al. 2004] A. Gerasimov, S. Kharchev, D. Lebedev, and S. Oblezin, "On a class of representations of the Yangian and moduli space of monopoles", preprint, 2004. arXiv math/0409031

[Ginzburg 1995] V. Ginzburg, "Perverse sheaves on a loop group and Langlands duality", preprint, 1995. arXiv alg-geom/9511007

[Kaledin and Verbitsky 2002] D. Kaledin and M. Verbitsky, "Period map for non-compact holomorphically symplectic manifolds", Geom. Funct. Anal. 12:6 (2002), 1265-1295. MR 2004e:32011 Zbl 1032.58009

[Levendorskii 1993] S. Z. Levendorskii, “On PBW bases for Yangians”, Lett. Math. Phys. 27:1 (1993), 37-42. MR 94b:17027 Zbl 0781.17004

[Losev 2012] I. Losev, "Isomorphisms of quantizations via quantization of resolutions", Adv. Math. 231:3-4 (2012), 1216-1270. MR 2964603 Zbl 06094077

[Lu and Yakimov 2008] J.-H. Lu and M. Yakimov, "Group orbits and regular partitions of Poisson manifolds”, Comm. Math. Phys. 283:3 (2008), 729-748. MR 2009i:53084 Zbl 1153.53059

[Lusztig 1981] G. Lusztig, "Green polynomials and singularities of unipotent classes", Adv. in Math. 42:2 (1981), 169-178. MR 83c:20059 Zbl 0473.20029

[Lusztig 1983] G. Lusztig, "Singularities, character formulas, and a $q$-analog of weight multiplicities", pp. 208-229 in Analysis and topology on singular spaces, II, III (Luminy, 1981), Astérisque 101, Soc. Math. France, Paris, 1983. MR 85m:17005 Zbl 0561.22013

[Mirković and Vilonen 2007] I. Mirković and K. Vilonen, "Geometric Langlands duality and representations of algebraic groups over commutative rings", Ann. of Math. (2) 166:1 (2007), 95-143. MR 2008m:22027 Zbl 1138.22013

[Mirković and Vybornov 2007] I. Mirković and M. Vybornov, "Quiver varieties and BeilinsonDrinfeld Grassmannians of type A", preprint, 2007. arXiv 0712.4160

[Namikawa 2001] Y. Namikawa, "A note on symplectic singularities", preprint, 2001. arXiv math/ 0101028

[Namikawa 2010] Y. Namikawa, "Poisson deformations of affine symplectic varieties, II", Kyoto J. Math. 50:4 (2010), 727-752. MR 2012b:14067 Zbl 1211.14040

[Namikawa 2011] Y. Namikawa, "Poisson deformations of affine symplectic varieties", Duke Math. J. 156:1 (2011), 51-85. MR 2012a:14023 Zbl 1208.14028

[Richardson 1992] R. W. Richardson, "Intersections of double cosets in algebraic groups", Indag. Math. (N.S.) 3:1 (1992), 69-77. MR 93b:20081 Zbl 0833.22001 
[Weyman 1989] J. Weyman, "The equations of conjugacy classes of nilpotent matrices", Invent. Math. 98:2 (1989), 229-245. MR 91g:20070 Zbl 0717.20033

Communicated by Edward Frenkel

Received 2013-01-10 Revised 2013-08-02 Accepted 2013-08-31

jkamnitz@math.toronto.edu Department of Mathematics, University of Toronto, 40 St. George Street, Toronto, ON M5S 2E4, Canada

bwebster@virginia.edu

Department of Mathematics, University of Virginia, 141 Cabell Drive, Charlottesville, VA 22904, United States

alex.weekes@utoronto.ca

Department of Mathematics, University of Toronto, 40 St. George Street, Toronto, ON M5S 2E4, Canada

oded.yacobi@sydney.edu.au

School of Mathematics and Statistics, University of Sydney, NSW 2006, Australia 


\section{Algebra \& Number Theory}

msp.org/ant

\section{EDITORS}

MANAGING EDITOR

Bjorn Poonen

Massachusetts Institute of Technology

Cambridge, USA

\author{
EDITORIAL BOARD CHAIR \\ David Eisenbud \\ University of California \\ Berkeley, USA
}

BOARD OF EDITORS

Georgia Benkart

Dave Benson

Richard E. Borcherds

John H. Coates

J-L. Colliot-Thélène

Brian D. Conrad

Hélène Esnault

Hubert Flenner

Edward Frenkel

Andrew Granville

Joseph Gubeladze

Roger Heath-Brown

Craig Huneke

Yujiro Kawamata

János Kollár

Yuri Manin

Barry Mazur

Philippe Michel

Susan Montgomery
University of Wisconsin, Madison, USA

University of Aberdeen, Scotland

University of California, Berkeley, USA

University of Cambridge, UK

CNRS, Université Paris-Sud, France

University of Michigan, USA

Freie Universität Berlin, Germany

Ruhr-Universität, Germany

University of California, Berkeley, USA

Université de Montréal, Canada

San Francisco State University, USA

Oxford University, UK

University of Virginia, USA

University of Tokyo, Japan

Princeton University, USA

Northwestern University, USA

Harvard University, USA

École Polytechnique Fédérale de Lausanne

University of Southern California, USA
Shigefumi Mori

Raman Parimala

Jonathan Pila

Anand Pillay

Victor Reiner

Peter Sarnak

Joseph H. Silverman

Michael Singer

Vasudevan Srinivas

J. Toby Stafford

Bernd Sturmfels

Richard Taylor

Ravi Vakil

Michel van den Bergh

Marie-France Vignéras

Kei-Ichi Watanabe

Efim Zelmanov

Shou-Wu Zhang
RIMS, Kyoto University, Japan

Emory University, USA

University of Oxford, UK

University of Notre Dame, USA

University of Minnesota, USA

Princeton University, USA

Brown University, USA

North Carolina State University, USA

Tata Inst. of Fund. Research, India

University of Michigan, USA

University of California, Berkeley, USA

Harvard University, USA

Stanford University, USA

Hasselt University, Belgium

Université Paris VII, France

Nihon University, Japan

University of California, San Diego, USA

Princeton University, USA

\section{PRODUCTION}

production@msp.org

Silvio Levy, Scientific Editor

See inside back cover or msp.org/ant for submission instructions.

The subscription price for 2014 is US $\$ 225 /$ year for the electronic version, and $\$ 400 /$ year ( $\$ 55$, if shipping outside the US) for print and electronic. Subscriptions, requests for back issues and changes of subscribers address should be sent to MSP.

Algebra \& Number Theory (ISSN 1944-7833 electronic, 1937-0652 printed) at Mathematical Sciences Publishers, 798 Evans Hall \#3840, c/o University of California, Berkeley, CA 94720-3840 is published continuously online. Periodical rate postage paid at Berkeley, CA 94704, and additional mailing offices.

ANT peer review and production are managed by EditFLOw ${ }^{\circledR}$ from Mathematical Sciences Publishers.

\section{PUBLISHED BY}

mathematical sciences publishers

nonprofit scientific publishing

http://msp.org/

(C) 2014 Mathematical Sciences Publishers 


\section{Algebra \& Number Theory}

Volume $8 \quad$ No. $4 \quad 2014$

The derived moduli space of stable sheaves

Kai Behrend, Ionut Ciocan-Fontanine, Junho Hwang and Michael Rose

Averages of the number of points on elliptic curves

Greg Martin, Paul Pollack and Ethan Smith

Noncrossed product bounds over Henselian fields

Timo Hanke, DanNy NeFtin and JaCk SONN

Yangians and quantizations of slices in the affine Grassmannian

Joel Kamnitzer, Ben Webster, Alex Weekes and Oded Yacobi

Equidistribution of values of linear forms on quadratic surfaces

OLIVER SARGENT

Posets, tensor products and Schur positivity

Vyjayanthi Chari, Ghislain Fourier and Daisuke SAGaKi

Parameterizing tropical curves I: Curves of genus zero and one

DAVID E. SPEYER

Pair correlation of angles between reciprocal geodesics on the modular surface

Florin P. Boca, Vicențiu Pașol, AleXandru A. Popa and AleXandru

ZAHARESCU

Étale contractible varieties in positive characteristic

ARMin HolschbaCh, JohanNes SCHMIDT and JaKob STIX 